\title{
ON OBSTRUCTION THEORY IN ORIENTABLE FIBER BUNDLES $\left({ }^{1}\right)$
}

\author{
BY \\ MARK MAHOWALD
}

\section{Introduction.}

1.1. There have been several efforts in recent years to describe a procedure for determining whether or not for a given fiber bundle $(E, B, p, F, G)$, in the sense of Steenrod [16], there exists a map $\phi: B \rightarrow E$, called a cross section, such that $p \phi$ is the identity. This paper represents another such effort. We will investigate obstructions to finding a cross section for a bundle with $(n-1)$-connected fiber $F$ such that $\sum_{i=1}^{2 n-1} \pi_{i}(F)$ is finitely generated and $\pi_{1}(B)$ acts trivially on the fiber. We will describe a general procedure which answers the question of the existence of a cross section if $B$ has cohomology dimension less than $2 n$. The procedure gives some information beyond this, but it is not clear now how useful this will be.

The general idea is similar to Hermann's in [6]. Formulas which generalize Liao's formula [9] for the secondary obstruction to a sphere bundle are also obtained. One of the features which enables higher obstruction classes to be identified is the grouping together of all obstructions of the "same level." Another feature is the investigation of only the prime parts of each obstruction. For example, if a homotopy group of $F$ is $Z_{4}$ in some dimension, then we look at the $Z_{2}$ parts of the sequence

$$
0 \rightarrow Z_{2} \rightarrow Z_{4} \rightarrow Z_{2} \rightarrow 0
$$

only.

In $\S 2$ the notion of a modified Postnikov tower (MPT) is introduced and its existence is proved in the case of interest (Theorem 2.2.5). In $\$ 3$ we develop some tools useful in evaluating the classes needed for the tower. $\$ 4$ contains a detailed computation of the tower for $n$-sphere bundles up to dimension $n+4$. This is sufficient to find cross sections of $n$-sphere bundles over $(n+6)$-dimensional complexes. In $\S 5$ we investigate the connection of a MPT with the obstruction question. The computations for $V_{n+m, m}$ bundles are contained in $\S 6$. The study is carried far enough to answer the question of the existence of a cross section if $B$ has cohomology dimensions less than $n+5$.

Received by the editors October 10, 1962.

(1) This research was supported by a grant from the U. S. Army Research Office (Durham). 
In $\$ 7$ some of the applications announced in [10] are given. In particular we prove

THEOREM 7.1.1. Let $M^{n}$ be a compact orientable n-manifold, $n>4$. If $n$ is even then $M^{n}$ immerses in $R^{2 n-2}$ iff $\bar{w}_{2} \cdot \bar{w}_{n-2}=0$; if $n$ is odd then $M^{n}$ immerses in $R^{2 n-2}$. ( $\bar{w}_{i}$ is the dual Stiefel-Whitney class.)

THEOREM 7.2.2. The following embeddings of projective spaces are possible.

(a) $k=3 \bmod 4, k>3$, then $P_{k} \subset R^{2 k-2}$.

(b) $k=4 s+i, s>0, s \neq 2^{j}, i=0,1$, or 2 , then $P_{k} \subset R^{2 k-3}$.

1.2. We will use the following conventions.

1.2.1. We use the symbol $G_{n}$ to refer to the space of all orientable $n$-planes in $R_{\infty}$.

1.2.2. In general we will let bold-faced German letters $\mathfrak{b}$ refer to bundles $(E, B, p, F, G)$. The only exceptions are the special bundles defined in 1.2.5. Let $B G$ be the classifying space of bundles with group $G$. Then each bundle $\mathfrak{b}$ has a classifying map $\mathfrak{b}: B \rightarrow B G$. In general, if the fiber and group are known from context, we write $\mathfrak{b}=(E, B, p)$. Note also that $B S O(n)=G_{n}$.

1.2.3. In $\S \S 4,6$ and 7 we will only consider $S O(n)$ bundles with one exception (1.2.5). The Stiefel-Whitney classes are cohomology classes in $H^{*}\left(G_{n} ; Z_{2}\right)$. We will identify $w_{2 i+1}$ with $\beta_{2} w_{2 i} \in H^{*}\left(G_{n} ; Z\right)$ where $\beta_{q}$ is the Bockstein coboundary associated with the sequence

$$
0 \rightarrow Z \rightarrow Z \rightarrow Z_{q} \rightarrow 0 .
$$

The Stiefel-Whitney classes of $\mathfrak{b}$ will be written $w_{i}(\mathfrak{b})$. Associated with $\mathfrak{b}$ will be $V_{n, m}$ bundles $\mathfrak{b}^{m}$.

1.2.4. Throughout this paper we require that the bundles are orientable. To be specific, we require that $\pi_{1}(B)$ act trivially on $H^{*}(F)$. This will always be a standing hypothesis with the one exception of 1.2.5.

1.2.5. We will use the symbol $s I=(s I, B, p)$ (or just $s I$ when the rest is clear) to mean a trivial $s$-plane bundle over $B$. The bundle $s H_{j}=\left(s H_{j}, P_{j}, p\right)$ will be the Whitney sum $s$ times of the nontrivial line bundle over $P_{j}$, real $j$-dimensional projective space. This bundle is not orientable if $s$ is odd and is the only nonorientable bundle which we will consider.

1.2.6. The set $B$ will be any connected $\mathrm{CW}$ complex. We suppose all our spaces have a fixed base point which we denote by *.

1.2.7. If $(E, B, p)$ is any fiber bundle in the sense of Steenrod [16] we can always suppose there exists a subset $M$ of $B$ and maps $\lambda: M \rightarrow E$ and $\lambda_{0}: M \subset B$ such that $p \lambda=\lambda_{0}$ and $\lambda$ is a homotopy equivalence. Indeed let $B^{\prime}$ be the mapping cylinder of $p: E \rightarrow B$. Let $\left(E^{\prime}, B^{\prime}, p^{\prime}\right)$ be the fiber bundle induced by $(E, B, p)$ from the canonical map $f: B^{\prime} \rightarrow B$. Then $E^{\prime}$ is homotopically equivalent with 
$E, B^{\prime}$ with $B$ and $E$ is embedded as a subset of $B^{\prime}$. Taking $M=E$ and dropping all the primes, we have the result.

1.2.8. The symbol $Z_{q}$ will mean $Z / q Z$ if $q \neq 0$ and $Z$ if $q=0 ; q$ will usually be a prime or 0 .

1.3. Let $K(J, n)$ be an Eilenberg-MacLane complex associated with $n$ and $J$, i.e., $K(J, n)$ has one nonzero homotopy group and that is $\pi_{n}(K(J, n))=J$. We will usually use $\alpha$ to designate the characteristic class of $K(J, n)$. According to Milnor [13], if $n>1$, there is a principal fiber bundle $\mathfrak{p}=(L K, K(J, n), K(J, n-1) \pi, K(J, n-1))$ such that $L K$ is acyclic.

1.3.1. By the space over $B$ with fiber $K(J, n-1)$ and $k$-invariant $a \in H^{n}(B ; J)$, we mean the fiber space induced by the map $f: B \rightarrow K(J, n)$, where $f^{*}(\alpha)=a$, from the fiber space $\mathfrak{p}$. We have the diagram

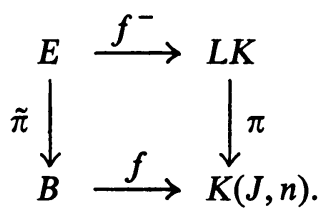

The following facts about this situation are well known.

1.3.2. $\tilde{\pi}^{*} a=0$.

1.3.3. In the sequence

$$
H^{n-1}(K(J, n-1)) \stackrel{\delta^{*}}{\rightarrow} H^{n}(E, K) \stackrel{\tilde{\pi}^{*}}{\leftarrow} H^{n}(B, *) \underset{j^{*}}{\simeq} H^{n}(B),
$$

we have $\delta^{*} \alpha=\tilde{\pi}^{*} j^{*-1} a$.

1.3.4. If $g: X \rightarrow B$, then there exists a map $g_{1}: X \rightarrow E$ such that $\tilde{\pi} g_{1}=g$ iff $g^{*} a=0$. We call such a mapping $g_{1}$, a lifting of $g$.

1.4. The possibility of generalizing the technique of [10] to the more general situation of $\S \S 2$ and 3 by using 1.2.7 was suggested to us by W. S. Massey. Many other improvements are due to him and we wish to express our thanks to him. We are also indebted to R. Schwarzenberger for sending us a preprint of [4] and calling our attention to the fact that obstruction theory could give information on embedding projective spaces. With his permission Theorem 7.2.1 is included here.

\section{Modified Postnikov tower.}

2.1. The grouping together of obstructions of a common level will be based on the notion of a regular spherical set.

Definition 2.1.1. Let $X$ be an $(n-1)$-connected space, $n>1$, such that $\pi_{k}(X)$ is finitely generated. A set $\mathscr{S}^{k}\left(X ; Z_{q}\right) \subset H^{k}\left(X ; Z_{q}\right)$ is called a regular spherical set if

(1) $k<2 n$; 
(2) for each $a \in \mathscr{S}^{k}\left(X ; Z_{q}\right)$, there exists a map $f_{a}: S^{k} \rightarrow X$ such that $f^{*}(a)$ generates $H^{k}\left(S^{k} ; Z_{q}\right)$;

(3) the set of homotopy classes of maps $\left\{\left[f_{a}\right]\right\} \quad a \in \mathscr{S}^{k}(X ; Z)$ is a linearly independent set in $\pi_{k}(X)$ considered as a $Z$ module and the set $\left\{\left[\bar{f}_{a}\right]\right\}_{a \in \mathscr{S}^{k}(X ; Z)} \cup$ $\left\{\left[\tilde{f}_{a}\right]\right\}_{a \in \mathscr{S} k_{\left(X: Z_{q}\right)}}$ is linearly independent over $Z_{q}$ where $\tilde{f}_{a}$ is the image of $f_{a}$ under $\pi_{k}(X) \rightarrow \pi_{k}(X) \otimes Z_{q}$.

(4) $\mathscr{S}^{k}\left(X ; Z_{q}\right)$ is not a proper subset of any set satisfying 1-3.

In general, regular spherical sets are not unique. It is also clear that they exist. The following theorem with its constructive proof gives some insight into just what these sets are.

THEOREM 2.1.2. Let $X$ be an $(n-1)$-connected space, $n>1$, such that $\pi_{k}(X)$ is finitely generated, $k<2 n$. Then for each prime $q$ and for $q=0$ there is $a$ regular spherical set $\mathscr{S}^{k}\left(X ; Z_{q}\right)$.

Proof. By the fundamental theorem of finitely generated Abelian groups, $\pi_{k}(X)$ is isomorphic to a direct sum of cyclic subgroups, either of infinite or prime power order. Let $a_{1}, \cdots, a_{t}$ be generators of these subgroups where $a_{i}$, $i \leqq s$, has infinite order while $a_{i}, i>s$ has finite order.

Each class $a_{i}$ induces a homomorphism $a_{i}^{*}: H^{k}(X ; Z) \rightarrow H^{k}\left(S^{k} ; Z\right)=Z$. Choose a representation $H^{k}\left(S^{k} ; Z\right)=Z$ and keep it fixed. Let $b_{1}^{\prime}, \cdots, b_{s}^{\prime}$ be independent generators of the free Abelian part of $H^{k}(X ; Z)$. Let $B$ be the subgroup generated by $\left\{b_{i}^{\prime}\right\}$ and let $Z^{s}=Z+\cdots+Z$ with $s$ summands. Define $\mathbf{a}: B \rightarrow Z^{s}$ by $\mathbf{a}=\left(a_{1}^{*}, \cdots, a_{s}^{*}\right)$. By the theorem of Serre [14], p. 108, a must be a monomorphism. Therefore we can find a basis $\left\{b_{i}\right\}$ for $B$ and $\left\{e_{i}\right\}$ for $Z^{s}$ such that $a\left(b_{i}\right)=d_{i} e_{i}$ where $d_{i} \mid d_{i+1}$. Let $\mathscr{S}^{k}(X ; Z)=\left\{b_{i} ; d_{i}=1\right\}$. Let $\left\{e_{i}^{\prime}\right\}$ be the standard basis for $Z^{s}$ and let $\mathfrak{p}=\left(p_{i j}\right)$ be the matrix such that $e_{i}^{\prime}=\sum_{j=1}^{s} p_{i j} e_{j}$. Now if $f_{j}$ is a function in the homotopy class $\sum_{i=1}^{s} a_{i} p_{i j}$, then $f_{j}^{*} b_{j}=d_{j} \alpha$ where $\alpha$ is a generator of $H^{k}\left(S^{k} ; Z\right)$. Since the number of the $d_{i}$ which are equal to one is an invariant of a, all four parts of the definition are satisfied for $\mathscr{S}^{k}(X ; Z)$.

Let $B_{q}$ be the image of $B$ under the homomorphism induced by $Z \rightarrow Z_{q}$. Let $T$ be the complementary vector subspace to $B_{q}$ of $H^{k}\left(X ; Z_{q}\right)$. If we identify $H^{k}\left(S^{k} ; Z_{q}\right)$ with $Z_{q}$, then each $a_{i}$ defines a linear functional $a_{i}^{*}$. We look at the restriction of each to $T$ and select a maximal linearly independent set $a_{i_{1}}^{*}, \cdots, a_{i .}^{*}$. Extend this collection to a basis of the dual of $T$ and construct the dual basis in $T$. The set $\mathscr{S}^{k}\left(X ; Z_{q}\right)$ consists of those classes in this basis which are dual to $a_{i_{1}}^{*}, \cdots, a_{i_{u}}^{*}$. These sets $\mathscr{S}^{k}\left(X ; Z_{q}\right)$ clearly satisfy the definition.

2.1.3. EXAMPLE. For any $(n-1)$-connected space $X, \mathscr{S}^{n}(X ; Z)$ is a basis for the free abelian part of $H^{n}(X ; Z)$ and $\mathscr{S}^{n}\left(X ; Z_{q}\right)$ is chosen so that $\mathscr{S}^{n}\left(X ; Z_{q}\right)$ $\cup \mathscr{S}^{n}(X ; Z)_{q}$ is a basis for $H^{n}\left(X ; Z_{q}\right)$.

2.2. Let $\mathfrak{b}=(E, B G, p)$ be the universal fiber bundle having a given structure group $G$ and a given $G$-space $F$ as fiber. Let $\mathfrak{b}^{\prime}=\left(E^{\prime}, B^{\prime}, p^{\prime}\right)$ be any other bundle having $F$ and $G$ as fiber and structure group. Then there is a bundle map 


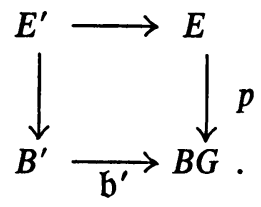

A necessary and sufficient condition that $\mathfrak{b}^{\prime}$ have a cross section is that there exists a map $\tilde{\mathrm{b}}^{\prime}: B^{\prime} \rightarrow E$ such that $p \tilde{\mathfrak{b}}^{\prime}=\mathfrak{b}^{\prime}$.

By 1.2.7 we have a space $M$ and maps $\lambda_{0}$ and $\lambda$ such that

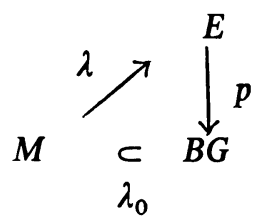

is commutative and $\lambda$ is a homotopy equivalence. Let $\tilde{\mathbf{b}}=(\tilde{M}, M, \tilde{p})$ be the bundle over $M$ induced by $\lambda_{0}$ from $\mathfrak{b}$. Clearly $\tilde{\mathbf{b}}$ is homotopically equivalent to the square of $\mathfrak{b}[16$, p. 49$]$ and the remarks above state that $\tilde{\mathfrak{b}}$ is universal for $F$ bundles having a cross section. This helps to motivate the next definition.

Definition 2.2.1. Consider the sequence

$$
H^{k}(\tilde{M}) \rightarrow H^{k}(F) \stackrel{\delta^{*}}{\rightarrow} H^{k+1}(\tilde{M}, F)
$$

We say $F$ is $t$-regular if there exists an $\mathscr{S}^{k}\left(F ; Z_{q}\right)$ such that $\delta^{*} \mathscr{S}^{k}\left(F ; Z_{q}\right)=0$ for all $k \leqq t$ and all primes $q$ or $q=0$. Note that if $t^{\prime}<t$ and $F$ is $t$-regular it is also $t^{\prime}$-regular.

Definition 2.2.2. Let $\mathscr{S}_{t}(F)=\bigcup_{q} \bigcup_{k=1}^{t} \mathscr{S}^{k}\left(F ; Z_{q}\right)$. By a representation of $\mathscr{S}_{t}(F)$ we mean a map $h: F \rightarrow Y$ where $Y=\prod_{q} \prod_{k=1}^{t} \prod_{a \in \mathscr{S}_{k}\left(F ; Z_{q}\right)} K_{a}\left(Z_{q}, k\right)$ and where $h^{*}\left(\alpha_{a}\right)=a$ for $\alpha_{a}$, the image of the characteristic class of $K_{a}\left(Z_{q}, k\right)$ in $H^{*}(Y)$.

When it is necessary to be more precise about $a \in \mathscr{S}_{t}(F)$ we write $a(q, k)$ for a class in $\mathscr{S}^{k}\left(F ; Z_{q}\right)$.

Definition 2.2.3. A modified Postnikov tower, MPT (of dimension $t$ ), for $\mathbf{b}=(E, B, p)$ is a tower

such that:

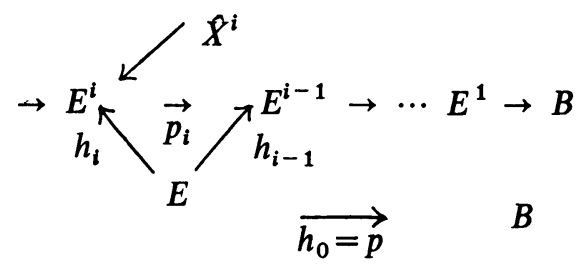

(1) The map $p_{i}: E^{i} \rightarrow E^{i-1}$ is a nontrivial fibering with fiber $\hat{X}^{i}=\prod_{a \in A} K_{a}\left(J_{a}, k_{a}\right)$ where $A$ is a finite index set and $J_{a}$ is either $Z$ or $Z_{q}$ for some prime $q$.

(2) The map $h_{i-1}: E \rightarrow E^{i-1}$ is a fibering with fiber $X^{i}$. 
(3) The space $X^{i}$ is $t_{i}$-regular where $\left(t \geqq t_{i}\right.$ and) $t_{i} \geqq n_{i}$, the dimension of the first nonzero homotopy group of $X^{i}$.

(4) The map $h_{i} \mid X^{i}: X^{i} \rightarrow \hat{X}^{i}$ is a representation of $\mathscr{S}_{t_{i}}\left(X^{i}\right)$.

2.2.4. In the above definition if $t_{i}=n_{i}$ for all $i$, then MPT is just the tower discussed in [10] for the universal $S O(n+m)$ bundle having $V_{n+m, m}$ as a fiber.

TheOREM 2.2.5. Let $G$ be arcwise connected; let $F$ be a G-space which is $(n-1)$-connected and for which $\pi_{j}(F)$ is finitely generated for $j \leqq t<2 n$; and let $\mathfrak{b}=(E, B G, p)$ be the universal $F$ bundle. Then $\mathfrak{b}$ has a finite MPT of dimension $t$.

Proof. The hypothesis that $G$ is arcwise connected implies $\boldsymbol{b}$ is orientable in the sense of $1.2 .4,[8]$. Let $\tilde{\mathbf{b}}=(\tilde{M}, M, \tilde{p})$ be the bundle defined above. Because $p \lambda=\lambda_{0}, \tilde{M}$ has a cross section $\rho: M \rightarrow \tilde{M}$ which we choose so that

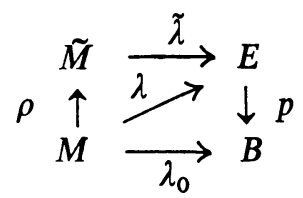

is a commutative diagram. Then $H^{*}(\tilde{M})=\operatorname{im} \tilde{p}^{*}+\operatorname{ker} \rho^{*}$. Consider the diagram

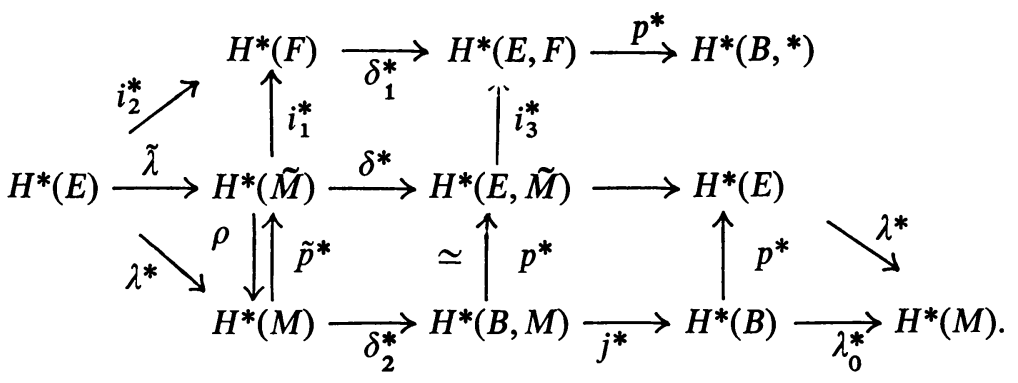

The isomorphism $p^{*}$ holds up to dimension $2 n$ since $(B, M)$ and $F$ are both $(n-1)$-connected. Suppose $F$ is $t_{1}$-regular and $\mathscr{S}_{t_{1}}(F)$ is given. The definition of $t_{1}$-regularity and the fact that $t_{1}<2 n$ implies that for each $a \in \mathscr{S}^{k}\left(F ; Z_{q}\right), k \leqq t_{1}$, there is a unique class in $H^{k}\left(\tilde{M} ; Z_{q}\right) \cap \operatorname{ker} \rho^{*}$ which maps onto $a$ under the restriction map $i_{1}^{*}: H^{*}(\tilde{M}) \rightarrow H^{*}(F)$. We identify $a$ with this class. Let $\mathbf{k}_{a}^{1}=j^{*} p^{*-1} \delta^{*} a$. Let $p_{1}: E^{1} \rightarrow B$ be the fiber space having

$$
\hat{X}^{1}=\prod_{q} \prod_{k=1}^{t_{1}} \prod_{a \in \mathscr{S}^{k}\left(F ; Z_{n}\right)} K_{a}\left(Z_{q}, k\right)
$$

as the fiber and $\left\{\mathbf{k}_{a}^{1}\right\}$ as the $k$-invariants. Then the map $p: E \rightarrow B$ can be lifted to a map $h_{1}: E \rightarrow E^{1}$ since the only obstruction to such a lifting will be $p^{*} \mathbf{k}_{a}^{1}=\lambda^{*-1}\left(\lambda_{0}^{*} j^{*}\right)\left(p^{*-1} \delta^{*}\right)(a)=0$. 
In showing that a choice of $h_{1}$ exists such that $h_{1} \mid F: F \rightarrow \hat{X}^{1}$ is a representation of $\mathscr{S}_{t_{1}}(F)$, we use the following lemma, which is well known.

LEMMA 2.2.8. In the notation of diagram 2.2.7, we have $p^{*} \mathbf{k}_{a}^{1}=\delta_{1}^{*} i_{1}^{*} a$, and $\delta_{1}^{*} i_{1}^{*} a=0$ iff $\mathbf{k}_{a}^{1}=0$.

We will now show that a choice of $h_{1}$ exists such that $h_{1} \mid F: F \rightarrow \hat{X}^{1}$ is a representation of $\mathscr{S}_{t_{1}}(F)$. First observe that if $\alpha_{a}$ is the image of the characteristic class of $K_{a}\left(Z_{q}, k\right)$ in $H^{*}\left(\hat{X}^{1}\right)$, then $h_{1}^{*} \alpha_{a}\left(h_{1}\right.$ is $\left.h_{1} \mid F\right)$ varies over $i_{2}^{*} H^{k}\left(E ; Z_{q}\right)$. Consider the following diagram:

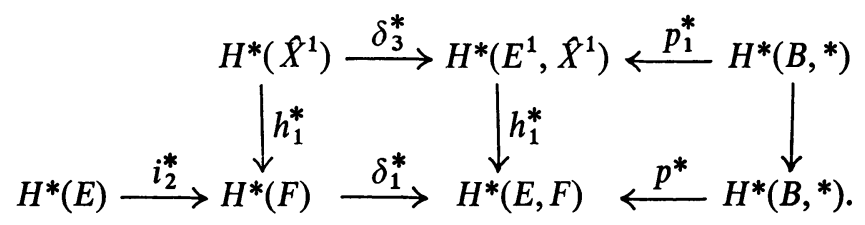

By 1.3.3, $p_{1}^{*} \mathbf{k}_{a}^{1}=\delta_{3}^{*} \alpha_{2}$ and by 2.2.8 $\delta_{1}^{*} a=p^{*} \mathbf{k}_{a}^{1}\left(i_{1}^{*} a=a\right)$. Therefore $h_{1}^{*} \alpha_{a}=a^{\prime}$ where $a-a^{\prime} \in i_{2}^{*} H^{k}\left(E ; Z_{q}\right)$. By a change of $h_{1}$, we then have $h_{1}^{*} \alpha_{a}=a$.

Now the proof of the theorem is completed by noting the inductive character of the definition. The fact that the tower is finite follows from the fact that $\pi_{n}\left(X^{2}\right)$ has fewer generators than $\pi_{n}(F)$ or the torsion part of $\pi_{n}\left(X^{2}\right)$ has fewer elements than the torsion part of $\pi_{n}(F)$. Indeed

$$
\pi_{n}\left(X^{2}\right) \stackrel{i_{*}}{\rightarrow} \pi_{n}(F) \stackrel{h_{1}^{*}}{\rightarrow} \pi_{n}\left(\hat{X}^{1}\right) \stackrel{\partial_{*}}{\rightarrow} \pi_{n-1}\left(X^{2}\right)=0
$$

and $h_{1}^{*}$ cannot be trivial since there must exist a $q$, prime or zero, such that $\mathscr{S}^{n}\left(F ; Z_{q}\right) \neq \phi(2.1 .3)$.

3. Determining the $\mathbf{k}$-invariants. The $\mathbf{k}^{1}$-invariants in the above construction seem readily identified as classes in $H^{*}(B)$ but the invariants at higher stages seem more complicated. In this section we will discuss a procedure which will enable us to make some computations.

3.1. Let $F$ be an (n-1)-connected space and let $h: F \rightarrow Y$ be a representation of $\mathscr{S}_{t}(F)$ for some $t$. Let $X$ be the fiber. We would like to be able to find $\mathscr{S}^{k}\left(X ; Z_{q}\right)$. Suppose the first nonzero homotopy group of $X$ occurs in dimension $n^{\prime}$. The hardest part of the definition to verify if $k>n^{\prime}$ is 2.1.1.3. In this paragraph we will prove Theorem 3.1.4 which gives a reasonable way to check for this condition. Of course $\mathscr{S}^{n^{\prime}}\left(X ; Z_{q}\right)$ is easily computed (2.1.3) for either $q$ zero or a prime.

Consider the sequence

$$
H^{j}(F) \stackrel{i^{*}}{\rightarrow} H^{j}(X) \stackrel{\hat{\delta}^{*}}{\rightarrow} H^{j+1}(Y) \stackrel{h^{*}}{\rightarrow} H^{j+1}(F)
$$

which is exact for $j<2 n-1$. A class $x_{j+1} \in H^{j+1}\left(Y ; Z_{q}\right)$ can be written in the form 


$$
x_{j+1}=\sum_{a \in \mathscr{S}_{t}(F)} \phi_{a} \alpha_{a}
$$

where $\phi_{a}$ is a primary cohomology operation $\phi_{a(q, k)}: H^{k}\left(Y ; Z_{q^{\prime}}\right) \rightarrow H^{k+1}\left(Y ; Z_{q}\right)$. (This is the usual representation of $H^{*}\left(K\left(\pi^{\prime}, k\right) ; \pi\right)$ as primary cohomology operations. If $\phi_{a\left(q^{\prime}, k\right)}$ is nonzero, then either $q$ and $q^{\prime}$ are the same or either one is zero.) Clearly $h^{*} x=\Sigma_{a \in \mathscr{S}_{t}(F)} \phi_{a}(a)$ and iff this is zero does there exist a class $x^{\prime} \in H^{j}(X)$ such that $\hat{\delta}^{*} x^{\prime}=x$.

Our next result gives a simple criterion for finding $x^{\prime}$ which satisfy 2.1.1.3. First we need a definition.

Definition 3.1.3. We say that an operation $\phi: H^{k}(X ; Z) \rightarrow H^{j+1}\left(X ; Z_{q}\right)$ is primitive if there is a map $g: S^{j} \rightarrow S^{k}$ such that $\phi$ is nontrivial in the complex $I^{j+1} \cup_{g} S^{k}$ where $I^{j+1}$ is a closed $(j+1)$-cell. An operation $\phi^{\prime}: H^{k}\left(X ; Z_{q}\right)$ $\rightarrow H^{j+1}\left(X ; Z_{q}\right)$ is primitive if: (1) the image of $\phi^{\prime}$ under the natural homomorphism $H^{*}\left(K\left(Z_{q}, k\right) ; Z\right) \rightarrow H^{*}\left(K(Z, k) ; Z_{q}\right)$ is primitive, or (2) if $\phi^{\prime}=\Delta_{q}$, the Bockstein coboundary of the sequence $0 \rightarrow Z_{q} \rightarrow Z_{q^{2}} \rightarrow Z_{q} \rightarrow 0$.

For example, $S q^{2^{i}}, i=0,1,2$, or 3 and $k>2^{i}$ are primitive [1]. Also it is not hard to show $\mathscr{P}_{3}^{1}$ is primitive. The next theorem justifies the definition.

THEOREM 3.1.4. Let $x \in H^{j+1}\left(Y ; Z_{q}\right)$ be represented in the form 3.1 .2 with $q \neq 0$ and suppose that for at least one $a^{\prime} \in \mathscr{S}_{t}(F), \phi_{a^{\prime}}$ is primitive. Then for any $x^{\prime} \in H^{j}(X)$ such that $\hat{\delta}^{*} x^{\prime}=x$, there exists a map $f: S^{j} \rightarrow X$ such that $f^{*} x^{\prime}$ generates $H^{j}\left(S^{j} ; Z_{q}\right)$.

Proof. Suppose $a^{\prime}\left(q^{\prime}, k\right)$ is such that $\phi_{a^{\prime}}$ is primitive. Clearly $q^{\prime}=0$ or $q$. Suppose $j>k$, the case $j=k\left(\phi_{a^{\prime}}=\Delta_{q}\right)$ being very similar. Let $A=I^{j+1} \cup_{g} S^{k}$ be the complex on which $\phi_{a^{\prime}}$ is nonzero. Choose generators of the cohomology of $A$,

$$
\beta_{1} \in H^{k}\left(A ; Z_{q^{\prime}}\right) \text { and } \beta_{2} \in H^{j+1}\left(A ; Z_{q}\right),
$$

such that $\phi_{a^{\prime}} \beta_{1}=\beta_{2}$. Let $M_{g}$ be the mapping cylinder of $g$. There is a natural embedding of $S^{j}$ in $M_{g}$. The space $A$ is homotopically equivalent to $M_{g} \cup C S^{j}$ where $C S^{j}$ is the cone over $S^{j}$, and the composite

$$
\text { 3.1.6 } H^{*}(A) \simeq H^{*}\left(M_{g} \cup C S^{j}\right) \rightarrow H^{*}\left(M_{g} \cup C S^{j}, C S^{j}\right) \rightarrow H^{*}\left(M_{g}, S^{j}\right)
$$

is an isomorphism in all dimensions greater than zero and which commutes with all natural cohomology operations. We identify the classes of 3.1.5 with their images under 3.1.6.

The space $M_{g}$ is homotopically equivalent with $S^{k}$ and so there exists a map $f: M_{g} \rightarrow F$ such that $f^{*}\left(a^{\prime}\right)$ generates $H^{k}\left(M_{g} ; Z_{q^{\prime}}\right)$. The map $f$ can be chosen so that $f:\left(M_{g}, S^{j}\right) \rightarrow(F, X)$ and so that $f^{*} h^{*} \alpha_{a}=0$ if $a \neq a^{\prime}$. Consider the sequences 


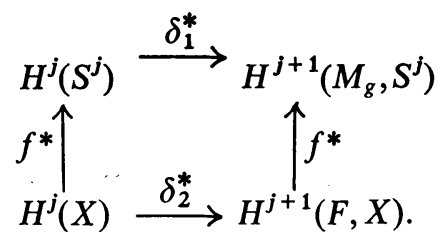

Let $x^{\prime}$ be such that $\hat{\delta} x^{\prime}=x$. Then $f^{*} \delta_{2}^{*} x^{\prime}=\Sigma \phi_{a} f^{*}\left(h^{*} \alpha_{a}\right)=\phi_{a^{\prime}} \beta_{1}=\beta_{2}$. Hence $f^{*} x^{\prime} \neq 0$ and since we are using a field for coefficients, we have the theorem.

3.1.7. For the applications we need one additional special case. Suppose $a^{\prime} \in \mathscr{S}^{k}\left(F ; Z_{2}\right), k \geqq 3$, and, if $f^{\prime}: S^{k} \rightarrow F$ is the map such that $f^{\prime *}\left(a^{\prime}\right)$ generates $H^{k}\left(S^{k} ; Z_{2}\right)$, then $2\left[f^{\prime}\right]=0$ where $\left[f^{\prime}\right]$ is the homotopy class of $f^{\prime}$. Let $x \in H^{k+3}\left(Y ; Z_{2}\right)$, represented in the form 3.1.2, be such that $\phi_{a^{\prime}}=S q^{2} S q^{1}$. Then for any $x^{\prime} \in H^{k+2}\left(X ; Z_{2}\right)$ such that $\hat{\delta}^{*} x^{\prime}=x$, there exists a map $f: S^{k+2} \rightarrow X$ such that $f^{*} x^{\prime}$ generates $H^{k+2}\left(S^{k+2} ; Z_{2}\right)$.

Proof. Let $B$ be the complex formed by adjoining $I^{k+1}$ to $S^{k}$ by a map of degree 2. If $k=5$ then $B$ can be realized as the eight skeleton of $V_{7,2}$ and hence the computations of [15] give the first few stable homotopy groups for all values of $k \geqq 3$ and in particular $\pi_{k+2}(B)=Z_{4}$. Let $g$ be a generator and let $A=I^{k+3} \cup_{g} B$. The first few homotopy groups of $A$ are: $\pi_{k}(A)=Z_{2}, \pi_{k+1}(A)=Z_{2}, \pi_{k+2}(A)=0$. Choose cohomology generators of $A$ as follows: $C_{1} \in H^{k}\left(A ; Z_{2}\right), S q^{1} C_{1} \in H^{k+1}\left(A ; Z_{2}\right)$, $C_{2} \in H^{k+3}\left(A ; Z_{2}\right)$. We claim that $S q^{2} S q^{1} C_{1}=C_{2}$. To see this consider the Postnikov tower:

$$
K\left(Z_{2}, k\right) \underset{l_{0} \uparrow_{A} l_{1}}{\stackrel{\omega}{l_{1}} .} P^{1}
$$

The first nonzero $k$-invariant is $S q^{2} \alpha$ since $S q^{2} C_{1}=0$. From the cohomology sequence of the pair $\left(P^{1}, K\left(Z_{2}, k+1\right)\right)$ we see easily that $S q^{2} S q^{1} \omega^{*} \alpha \neq 0$. This class is not a higher $k$-invariant since $\pi_{k+2}(A)=0$ and hence $S q^{2} S q^{1} C_{1}$ $=S q^{2} S q^{1} l_{1}^{*} \omega^{*} \alpha \neq 0$.

Now let $M_{g}$ be the mapping cylinder of $g: S^{k+2} \rightarrow B$, the map used to define $A$. As in 3.1.6 we see that $H^{*}(A) \simeq H^{*}\left(M_{g}, S^{k+2}\right)$ and we identify the generators of these two groups. Let $j^{*}: H^{*}\left(M_{g}, S^{k+2}\right) \rightarrow H^{*}\left(M_{g}\right)$ be the usual map. Let $f^{\prime}: S^{k} \rightarrow F$ be the map of hypothesis. Since $2\left[f^{\prime}\right]=0$, classical obstruction theory gives an extension of $f^{\prime}$ to a map $f: M_{g} \rightarrow F$. This map can be chosen to satisfy all the requirements made on $f$ in the last paragraph of the proof 3.1.4. Then the argument of that paragraph applies to this situation and completes the proof.

3.2. In 3.1 we investigated spherical sets. In this paragraph we will investigate how to find the $k$-invariants which go with spherical sets determined in the fashion of 3.1. We will work with a fixed prime $q$. 
Let

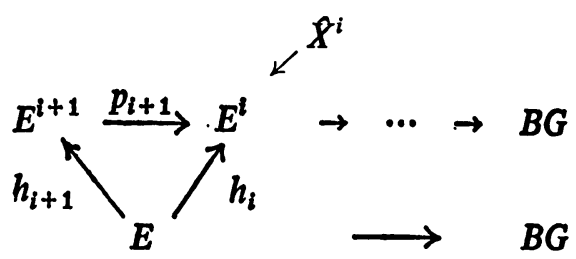

be an MPT for $\mathfrak{b}$, the universal bundle of 2.2 , for some dimension less than $2 n$ where the fiber $F$ is $(n-1)$-connected.

Let $\hat{X}^{i}=\prod_{q} \prod_{k=1}^{t_{i}} \prod_{a \in \mathscr{S}^{k}\left(X^{i} ; Z_{q}\right)} K_{a}\left(Z_{q}, k\right)$ and let $\mathbf{k}_{a}^{i}$ be the respective $k$-invariants. For $a \in \mathscr{S}^{k}\left(X^{i} ; Z_{q}\right), \mathbf{k}_{a}^{i} \in H^{k+1}\left(E^{i-1} ; Z_{q}\right)$.

Suppose, as an induction hypothesis, we have $\lambda_{i-1}: M \subset E^{i-1}$ where $M$ is the space discussed in 2.2. Consider the diagram:

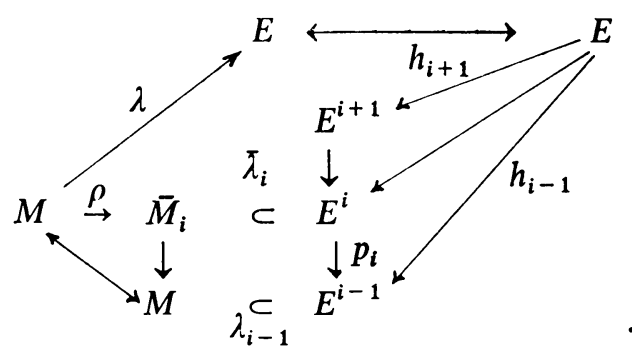

In this diagram $\bar{M}_{i}$ is the fiber space over $M$ induced by $\lambda_{i-1}$ from $p_{i}: E^{i} \rightarrow E^{i-1}$. Since $\lambda$ is a homotopy equivalence, we can require that $\lambda_{i-1}$ and $h_{i-1}$ be homotopically the same map and hence $\lambda_{i-1}^{*} \mathbf{k}_{a}^{i}=0$. Therefore $\bar{M}_{i}$ is homeomorphic to $M \times \hat{X}^{i}$ and $\rho$ is a cross section chosen so that $h_{i} \lambda=\lambda_{i} \rho$. For the next stage we take $\lambda_{i}=\bar{\lambda}_{i} \rho$. The new $k$-invariants, $\mathbf{k}^{i+1} \in H^{*}\left(E^{i}\right)$ will be classes in $\operatorname{ker} h_{i}^{*}=\operatorname{ker}\left(\rho^{*} \lambda_{i}^{*}\right)$.

These invariants arise from the fibering $h_{i}: E \rightarrow E^{i}$ having $X^{i+1}$ as the fiber. Let $\tilde{M}_{v}, v=i$ or $i+1$, be the space induced by

$$
\begin{array}{llll} 
& \tilde{M}_{v} & \stackrel{\lambda}{\subset} & E \\
\tilde{h}_{v-1} & \downarrow & & \downarrow \\
& M & \underset{\lambda_{v-1}}{\subset} & E^{v-1}
\end{array}
$$

We have a fibering $h_{i}: X^{i} \rightarrow \hat{X}^{i}$ and this induces a fibering $h_{i-1}: \tilde{M}_{i} \rightarrow \bar{M}_{i}$. Using the cross section $\rho: M \rightarrow \bar{M}_{i}$ we have $\tilde{\rho}: \tilde{M}_{i+1} \subset \tilde{M}_{i}$.

As data for the next theorem consider the sequence,

$$
H^{j-1}\left(X^{i}\right) \stackrel{i^{*}}{\rightarrow} H^{j-1}\left(X^{i+1}\right) \stackrel{\hat{\delta}^{*}}{\rightarrow} H^{j}\left(\hat{X}^{i}\right) \stackrel{h_{i}^{*}}{\rightarrow} H^{j}\left(X^{i}\right),
$$

analogous to 3.1.1. Let $a \in \mathscr{S}^{j-1}\left(X^{i+1} ; Z_{q}\right)$ and suppose $\hat{\delta}^{*} a \neq 0$. 
THEOREM 3.2.5. In the notation of 3.2.2. and 3.2.4, we have that if $X^{i+1}$ is (j-1)-regular, then $i^{*} \bar{\lambda}_{i}^{*} \mathbf{k}_{a}^{i+1}=\hat{\delta}^{*} a$ where $i: \hat{X}^{i} \subset \bar{M}_{i}$.

Proof. Consider the diagram

3.2.6

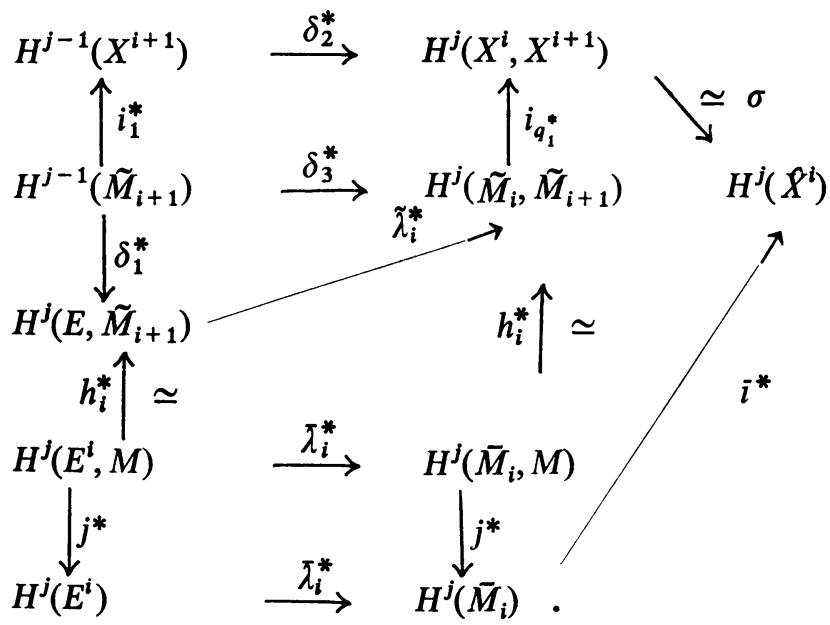

The isomorphism $\sigma$ is the composite

$$
H^{j}\left(X^{i}, X^{i+1}\right) \stackrel{h_{i}^{*}}{\simeq} H^{j}\left(\hat{X}^{i}, *\right) \rightarrow H^{j}\left(\hat{X}^{i}\right)
$$

and hence the map $\hat{\delta}^{*}$ is just $\sigma \delta_{2}^{*}$. The diagram is commutative and the left column serves to define $\mathbf{k}_{a}^{i+1}$. Recall that $a$ is identified with a class $a \in H^{j-1}\left(\tilde{M}_{i+1}\right)$ such that $i_{1}^{*} a=a$. Now $\sigma i_{1}^{*} \delta_{3}^{*} a=\hat{\delta}^{*} a$ and $\sigma i_{1}^{*} \delta_{3}^{*} a=i^{*} \lambda_{i}^{*} \mathbf{k}_{a}^{i+1}$ which completes the theorem.

3.3. Theorems 3.1.4 and 3.2.5 form the basis of our computation. Using 3.1.4 we find a class $x \in H^{j}\left(\hat{X}^{i}\right)$ which is the image of a class in a spherical set. To find the $k$-invariant associated with this $x$ we must find a class in $H^{j}\left(\bar{M}_{i}\right)$ such that it restricts to $x$ under $i^{*}$ but extends to a class in $H^{j}\left(E^{i}\right)$. Since each $k$-invariant is also in $\operatorname{ker} h_{i}^{*}$ and $\operatorname{ker} h_{i}^{*}=\operatorname{ker}\left(\rho^{*} \lambda_{i-1}^{*}\right)$ we will find such classes in $\operatorname{ker} \rho^{*}$.

By the Künneth formula $H^{*}\left(\bar{M}_{i} ; Z_{q}\right) \simeq H^{*}\left(M ; Z_{q}\right) \otimes H^{*}\left(\hat{X}^{i} ; Z_{q}\right)$. We can choose this representation so that in dimension $j, \operatorname{ker} \rho^{*} \cong \Sigma_{\mu=1}^{j} H^{j-\mu}(M) \otimes H^{\mu}\left(\hat{X}^{j}\right)$ and we identify these groups. Recall that $\hat{X}^{i}=\prod_{q} \prod_{k} \prod_{a \in S^{k}\left(X^{i} ; Z_{q}\right)} K_{a}\left(Z_{q}, k\right)$ and we let $\alpha_{a}$ be the image in $H^{*}\left(\hat{X}^{i}\right)$ of the characteristic class of $K_{a}$. Since each $\alpha_{a}$ is of dimension at least $n$ but of dimension less than $2 n$ we have, for each class $x_{\mu} \in H^{\mu}\left(\hat{X}^{i} ; Z_{q}\right), \mu<2 n$, that a relation such as 3.1 .2 holds. Hence a class $\sigma \in H^{j}\left(\bar{M}_{i} ; Z_{q}\right) \cap \operatorname{ker} \rho^{*}$ is a linear combination of classes $m_{j-\mu} \otimes x_{\mu}$ where $m_{v} \in H^{v}\left(M ; Z_{q}\right)$.

Definition 3.3.1. Let $\mathrm{e}=\left(e_{a}\right), a \in \mathscr{S}_{t}\left(X^{i}\right)$, where $e_{a(v, k)} \in H^{k+1}\left(E^{i-1} ; Z_{v}\right)$ and let $m_{j-\mu} \otimes x_{\mu} \in H^{j}\left(\bar{M}_{i+1} ; Z_{q}\right) \cap \operatorname{ker} \rho^{*}, j<2 n$. Then by $\left(m_{j-\mu} \otimes x_{\mu}\right)(e)$ we mean 
$(-1)^{j-\mu}\left(\lambda_{i-1}^{*-1} m_{j-\mu}\right) \cdot \Sigma_{a \in \mathscr{S}_{t}\left(X^{l}\right)} s \phi_{a} e_{a} \in H^{j+1}\left(E^{i-1} ; Z_{q}\right)$ where $s \phi_{a}$ maps onto $\phi_{a}$ under suspension. (Since $j-\mu<n, \lambda_{i-1}^{*}$ is an isomorphism.) By linearity this defines $\sigma(\mathbf{e})$.

The next lemma motivates the above definition. As data for it consider the diagram

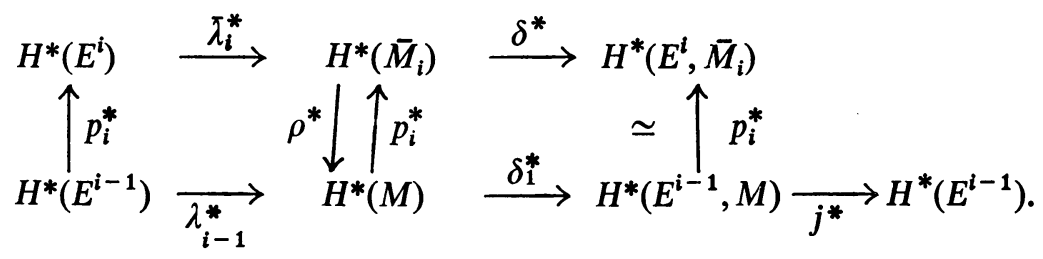

LEMMA 3.3.3. With the maps defined as in 3.3 .2 and letting $\mathbf{k}^{i}=\left(\mathbf{k}_{a}^{i}\right)$, we have $j^{*} p_{i}^{*-1} \delta^{*} \sigma=\sigma\left(\mathbf{k}^{i}\right)$.

Proof. It is sufficient to prove the formula for $m_{j-\mu} \otimes x_{\mu}$. Since $m_{j-\mu}$ is in $\operatorname{im} \lambda_{i}^{*}, \delta^{*}\left(m_{j-\mu} \otimes x_{\mu}\right)=(-1)^{j-\mu} m_{j-\mu} \cdot \delta^{*} x_{\mu}$, where $m_{j-\mu}$ is identified with its preimage in $H^{*}\left(E^{i}\right)$. Now $p_{i}^{*-1}\left(m_{j-\mu} \cdot \delta^{*} x_{\mu}\right)=\lambda_{i-1}^{*-1} m_{j-\mu} \cdot p_{i}^{*-1} x_{\mu}$. The proof is completed by noting $j^{*} p_{i}^{*-1} \delta^{*} \phi_{a} \alpha_{a}=\phi_{a} \mathbf{k}_{a}^{i}$ (1.3.3).

We are now ready to state and prove the main result of this paragraph.

THEOREM 3.3.4. Suppose $h_{i-1}^{*}$ is an epimorphism in dimension $j$. Then for $\sigma \in H^{j}\left(\bar{M}_{i} ; Z_{q}\right) \cap \operatorname{ker} \rho^{*}$, there exists a $v \in H^{j}\left(E^{i} ; Z_{q}\right) \cap \operatorname{ker} h_{i}^{*}$ such that $\bar{\lambda}_{i}^{*} v=\sigma$ iff $\sigma\left(\mathbf{k}^{i}\right)=0$.

Proof. Suppose $\sigma \in H^{j}\left(\bar{M}_{i} ; Z_{q}\right) \cap \operatorname{ker} \rho^{*}$ and $\sigma\left(\mathbf{k}^{i}\right)=0$. Then using diagram 3.3.2 and Lemma 3.3.3 we have $j^{*} p_{i}^{*-1} \delta^{*} \sigma=0$. Suppose $\delta^{*} \sigma \neq 0$. Then, by exactness, there exists a class $\sigma^{\prime} \in H^{j}(M)$ such that $p_{i}^{*} \delta_{1}^{*} \sigma^{\prime}=\delta^{*} \sigma$ and, in particular, $\delta_{1}^{*} \sigma^{\prime} \neq 0$. But by diagram 3.2.2 we see that $\lambda_{i-1}$ and $h_{i-1}$ are essentially the same map and $\lambda_{i-1}^{*}$ being an epimorphism implies $\delta_{1}^{*}$ is trivial. Hence $\delta_{1}^{*} \sigma^{\prime}=0$. Therefore $\sigma\left(\mathbf{k}^{i+1}\right)=0$ implies $\delta^{*} \sigma=0$ and, hence, there is a $v$ such that $\lambda_{i}^{*} v=\sigma$. Clearly $v \in \operatorname{ker} h_{i}^{*}$, since $\rho^{*} \sigma=0$ and $\operatorname{ker} h_{i}^{*}=\operatorname{ker}\left(\rho^{*} \lambda_{i}^{*}\right)$.

Conversely, if there exists such a $v$, then $\delta^{*} \sigma=0$ which implies $\sigma\left(\mathbf{k}^{l}\right)=0$.

3.4. The strongest tool for our computations is the next theorem. The statement and proof will be based on this diagram:

$$
H^{k}\left(E^{i}\right) \stackrel{\lambda_{i}^{*}}{\rightarrow} H^{k}\left(\bar{M}_{i}\right) \stackrel{\delta^{*}}{\rightarrow} H^{k+1}\left(E^{i}, \bar{M}_{i}\right) \stackrel{p_{i}^{*}}{\stackrel{\leftarrow}{\leftarrow}} H^{k+1}\left(E^{i-1}, M\right) \stackrel{j^{*}}{\rightarrow} H^{k+1}\left(E^{i-1}\right)
$$

3.4 .1

$$
\begin{array}{cc} 
& \\
\downarrow^{*} & H^{k+1}\left(E^{i-1}, *\right) \\
H^{k-1}\left(X^{i+1}\right) \stackrel{\delta^{*}}{\rightarrow} H^{k}\left(\hat{X}^{i}\right) \underset{h_{i}^{*}}{\rightarrow} H^{k}\left(X^{i}\right) & \stackrel{\delta_{2}^{*}}{\rightarrow} H^{k+1}\left(E, X^{i}\right) .
\end{array}
$$


THEOREM 3.4.2. Suppose $X^{i+1}$ is $j-2$-regular, $h_{i-1}^{*}$ is an epimorphism, and $\lambda_{i}^{*}$ is a monomorphism. Let $\mathbf{k}=\left(k_{1}, \cdots, k_{s}\right)$ be a collection of elements in $H^{j}\left(E^{i} ; Z_{q}\right), q \neq 0$ fixed, such that:

(1) The collection $i^{*} \lambda_{i}^{*} k_{v}$ is linearly independent.

(2) If $i^{*} \lambda_{i}^{*} k_{v}=x$ where $x$ is as defined by 3.1.2, then for at least one $a, \phi_{a}$ is primitive (3.1.4 applies) or 3.1.7 applies.

(3) Let $\mathscr{A}_{q}^{\mu}$ be the $\mu$-dimensional component of the Steenrod algebra. Then the vector subspace of $H^{j-1}\left(X^{i+1}\right)$ generated by $\bigcup_{u=1}^{j} \mathscr{A}_{q}^{\mu} H^{j-1-\mu}\left(X^{i+1}\right)$ has codimension $s$ in $H^{j-1}\left(X^{i+1}\right)$.

(4) $\bar{\lambda}_{i}^{*} k_{v} \in \operatorname{ker} \rho^{*}$.

Then there is a $\mathscr{S}^{j-1}\left(X^{i+1} ; Z_{q}\right)$ such that $\mathbf{k}$ is the collection $\left(\mathbf{k}_{a}^{i+1}\right), a \in \mathscr{S}^{j-1}\left(X^{i+1} ; Z_{q}\right)$.

Remark. Condition (3) seems complicated but if $j$ is only a few dimensions above the dimension of the first nonzero homotopy group it is easily checked. In addition note that if $s^{\prime}$ is the minimum number of generators of the $q$-primary part of $\pi_{j-1}\left(X^{i+1}\right)$, then $s \leqq s^{\prime}$ and so (3) is satisfied whenever $s=s^{\prime}$.

Proof. First observe that $j^{*} p_{i}^{*-1} \delta^{*} \alpha_{a}=\mathbf{k}_{a}^{i}$ (by 1.3.3). Now using 2.2.8 we have $h_{i-1}^{*} j_{1}^{*} p_{i}^{*-1} \delta^{*} \alpha_{a}=\delta_{2}^{*} h_{i}^{*} i^{*} \alpha_{a}$ since $h_{i}^{*} i^{*} \alpha_{a}=a\left(i_{1}^{*} a\right.$ in 2.2.8). Therefore $\delta_{2}^{*} h_{i}^{*} i^{*} \lambda_{i} k_{v}$ $=0$. From the following diagram

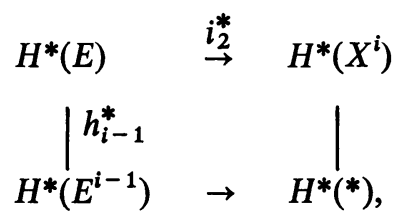

we see immediately that $h_{i-1}^{*}$ being an epimorphism implies $i_{2}^{*}$ is trivial and therefore $\delta_{2}^{*}$ is a monomorphism. Hence $h_{i}^{*} i^{*} \lambda_{i}^{*} k_{v}=0$ or there exists $a_{v} \in H^{j-1}\left(X^{i+1}\right)$ such that $\delta^{*} a_{v}=i^{*} \lambda_{i}^{*} k_{v}$. We claim $\left\{a_{v}\right\}=\mathscr{S}^{j-1}\left(X^{i+1} ; Z_{q}\right)$. Theorem 3.1 .4 proves 2.1.1.3. Parts (1) and (2) are clear, while (4) is an easy consequence of (3) and 2.1.1.3. Finally since $\bar{\lambda}_{i}^{*}$ is a monomorphism 3.2 .5 proves that $k_{j}=\mathbf{k}_{a_{j}}^{i+1}$.

4. To illustrate how these results are used to make computations consider the universal example for $n$-sphere bundles, $n \geqq 5 ; \mathfrak{g}=\left(E, G_{n+1}, p\right)$ where $G_{n+1}$ is $B S O(n+1)$. In this section we will not use 3.4 .2 since this would hide the details. Rather we go more or less directly to the definition using 3.1.4, 3.2.5 and 3.3.4 to find the $k$-invariants. In our later applications ( $\S 6)$ we will use 3.4.2 and this will shorten the computations considerably.

4.1. The generators of $H^{n}\left(S^{n} ; Z\right)$ satisfy 2.1.1.3 and we choose one of them $a \in \mathscr{S}^{n}\left(S^{n} ; Z\right)$. If $\chi$ is the Euler class we can suppose $a$ is chosen so that $\delta^{*} a=p^{*} \chi$ where $\delta^{*}$ is the coboundary in the sequence of the pair $\left(E, S^{k}\right)$. The first stage of MPT is 
4.1.1

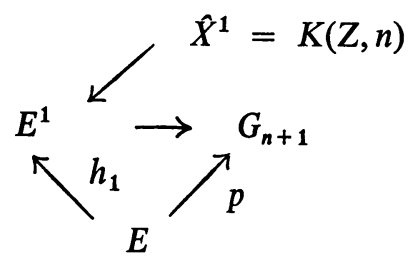

with $\mathbf{k}_{a}^{1}=\chi$.

4.2. To compute the second stage for the tower of dimension $n+3$, we need $\mathscr{S}^{k}\left(X^{2} ; Z_{q}\right)$ for $n<k \leqq n+3$. Consider the sequence

$$
\begin{aligned}
H^{k}\left(S^{n}\right) \stackrel{i^{*}}{\rightarrow} H^{k}\left(X^{2}\right) \stackrel{\delta^{*}}{\rightarrow} H^{k+1}\left(S^{n}, X^{2}\right) & \uparrow \simeq h_{1}^{*} \\
& H^{k+1}(K(Z, n), *) \simeq H^{k+1}(K(Z, n)) .
\end{aligned}
$$

Clearly $\delta^{*}$ is an isomorphism for $n<k \leqq n+4$. Hence :

$$
\begin{array}{ll}
H^{n+1}\left(X^{2} ; Z_{2}\right)=Z_{2} . & \text { generated by } a_{1}, \text { where } \delta^{*} a_{1}=h_{1}^{*} S q^{2} \alpha ; \\
H^{n+2}\left(X^{2} ; Z\right)=Z_{2}, & \text { generated by } a_{2}, \text { where } \delta^{*} a_{2}=h_{1}^{*} \beta_{2} S q^{2} \alpha ; \\
H^{n+3}\left(X^{2} ; Z_{2}\right)=Z_{2}, & \text { generated by } a_{3}, \text { where } \delta^{*} a_{3}=h_{1}^{*} S q^{4} \alpha ; \\
H^{n+3}\left(X^{2} ; Z_{3}\right)=Z_{3}, & \text { generated by } a_{4}, \text { where } \delta^{*} a_{4}=h_{1}^{*} \mathscr{P}{ }_{3}^{1} \alpha .
\end{array}
$$

Theorems 3.1.4 and 3.1.7 imply

$$
\begin{aligned}
& \mathscr{S}^{n+1}\left(X^{2} ; Z_{2}\right)=\left\{a_{1}\right\} \\
& \mathscr{S}^{n+3}\left(X^{2} ; Z_{2}\right)=\left\{a_{3}\right\} \\
& \mathscr{S}^{n+3}\left(X^{2} ; Z_{3}\right)=\left\{a_{4}\right\}
\end{aligned}
$$

and all other regular spherical sets are empty.

Consider the diagram

4.2.4

$$
\begin{aligned}
& H^{k+1}\left(E^{1}, \bar{M}_{1}\right) \stackrel{j_{1}^{*}}{\rightarrow} H^{k+1}\left(E^{1}\right) \stackrel{\lambda_{1}^{*}}{\rightarrow} H^{k+1}\left(\bar{M}_{1}\right) \stackrel{\delta^{*}}{\rightarrow} H^{k+2}\left(E^{1}, \bar{M}_{1}\right) \\
& \bigsqcup^{k}(K(Z, n)) H^{*+2}\left(G_{n+1}, M\right) \underset{j^{*}}{i^{*}} H^{k+2}\left(G_{n+1}\right) .
\end{aligned}
$$

4.2.5. First observe that $j_{1}^{*}=0$ if $k<2 n$ and that $h_{1}^{*}\left(h_{1}\right.$ is defined in 4.1.1) is an epimorphism. Suppose $k=n+1$. By 3.2 .5 we have $i^{*} \lambda_{1}^{*} \mathbf{k}_{a_{1}}^{2}=S q^{2} \alpha$ where $\alpha$ is the characteristic class of $K(Z, n)$. We identify $\alpha$ with the class in $H^{*}\left(\bar{M}_{1}\right) \cap \operatorname{ker} \rho^{*}$ which restricts to it (cf. 3.2.2). Since $S q^{2} \chi+w_{2} \cdot \chi=0, \sigma(\chi)=0$, where $\sigma=w_{2} \otimes \alpha+1 \otimes S q^{2} \alpha$. Therefore 3.3.4 implies $\mathbf{k}_{a_{1}}^{2}$ is the unique (since $j_{1}^{*}=0$ ) class such that $\bar{\lambda}_{1}^{*} \mathbf{k}_{a_{1}}^{2}=\sigma$. 
4.2.6. Using $S q^{4} \chi+w_{4} \cdot \chi=0$, as we used the similar formula for $S q^{2}$, we see that $\bar{\lambda}_{1}^{*} \mathbf{k}_{a_{3}}^{2}=w_{4} \otimes \alpha+1 \otimes S q^{4} \alpha$.

4.2.7. Finally if $n=0 \bmod 2,2 \chi=0$ and this implies $\mathscr{P}_{3}^{1} \chi=0$. In this case $\lambda_{1}^{*} \mathbf{k}_{a_{4}}^{2}=1 \otimes \mathscr{P P}_{3}^{1} \alpha$. If $n=1 \bmod 2$ then $\mathscr{P}_{3}^{1} \chi=\left(p_{1}\right)_{3} \cdot(\chi)_{3}$, where $p_{1}$ is the first Pontrjagin class, and we have $\lambda_{1}^{*} \mathbf{k}_{a_{4}}^{2}=1 \otimes \mathscr{P}_{3}^{1} \alpha+\left(p_{1}\right)_{3} \otimes \alpha$.

The second stage of MPT is

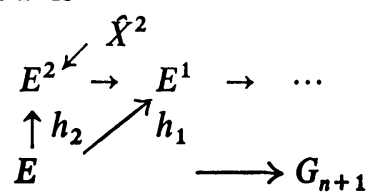

where

$$
\widehat{X}^{2}=K_{1}\left(Z_{2}, n+1\right) \times K_{3}\left(Z_{2}, n+3\right) \times K_{4}\left(Z_{3}, n+3\right) \text { d }\left(K_{i}=K_{a_{i}}\right) .
$$

4.3. For the third stage consider the sequence

$$
H^{k}\left(X^{2}\right) \stackrel{i^{*}}{\rightarrow} H^{k}\left(X^{3}\right) \stackrel{\hat{\delta}^{*}}{\rightarrow} H^{k+1}\left(\hat{X}^{2}\right) \stackrel{h_{2}^{*}}{\rightarrow} H^{k+1}\left(X^{2}\right)
$$

where $n<k \leqq n+5$. It is easy to check that $h_{2}^{*}$ is onto and, hence, $\hat{\delta}^{*}$ is a monomorphism. In addition $H^{k}\left(X^{3}\right) \simeq \operatorname{ker} h_{2}^{*}$ (in dimension $\left.k+1\right)$. Using $Z_{2}$ for coefficients and letting $x$ be as in 3.1.2, we have $h_{2}^{*} x=\phi_{a_{1}}\left(\hat{\delta}_{1}^{*-1} S q^{2} \alpha\right)+\phi_{a_{2}}\left(\hat{\delta}_{1}^{*-1} S q^{4} \alpha\right)$. This is zero iff $\phi_{a_{1}} S q^{2} \alpha+\phi_{a_{2}} S q^{4} \alpha=0$ in $K(Z, n)=\hat{X}^{1}$.

Theorem 3.1.4 asserts that if $h_{2}^{*} x=0$ and either $\phi_{a_{i}}, i=1$ or 2 , is primitive then $\hat{\delta}^{*-1} x \in \mathscr{S}^{k}\left(X^{3} ; Z_{2}\right)$. Since $S q^{1} S q^{1} \alpha=0, S q^{2} S q^{2} \alpha=0$ and $S q^{1} S q^{4} \alpha$ $+\left(S q^{2} S q^{1}\right) S q^{2} \alpha=0$, we have

$$
\begin{array}{ll}
b_{1} \in S^{n+2}\left(X^{3} ; Z_{2}\right), & \hat{\delta}^{*} b_{1}=S q^{2} \alpha_{1}, \\
b_{2} \in S^{n+3}\left(X^{3} ; Z_{2}\right), & \hat{\delta}^{*} b_{2}=S q^{1} \alpha_{3}+\left(S q^{2} S q^{1}\right) \alpha_{1} .
\end{array}
$$

By direct computation we have

$$
\left(S q^{2}+w_{2} \cdot\right)\left(S q^{2}+w_{2} \cdot\right) \alpha=0,
$$

$$
S q^{1}\left(S q^{4}+w_{4} \cdot\right) \alpha+\left(S q^{2} S q^{1}+w_{3} \cdot\right)\left(S q^{2}+w_{2} \cdot\right) \alpha=0 .
$$

Since $j_{1}^{*}$ is zero in 4.2 .4 these computations show

LEMMA 4.3.4.

$$
\begin{aligned}
\left(S q^{2}+w_{2} \cdot\right) \mathbf{k}_{a_{1}} & =0 \\
s S q^{1} \mathbf{k}_{a_{3}}+\left(S q^{2} S q^{1}+w_{3} \cdot\right) \mathbf{k}_{a_{1}} & =0.1
\end{aligned}
$$


Proof. Using diagram 4.2.4 we see that $\bar{\lambda}_{1}^{*}$ applied to the left side of an equation of 4.3.4 is just the left side of the corresponding equation of 4.3.3.

Now consider the sequence analogous to 4.2 .4 but for $E^{2}$. In this setting $j_{1}^{*}$ is again an isomorphism for $k \leqq n+3$. Hence knowing $\lambda_{1}^{*} \mathbf{k}_{b_{i}}$, determines uniquely $\mathbf{k}_{b_{i}}$. The equations of 4.3 .4 together with 3.2 .5 yield

$$
\begin{aligned}
& \lambda_{2}^{*} \mathbf{k}_{b_{1}}=1 \otimes S q^{2} \alpha_{1}+w_{2} \otimes \alpha_{1}, \\
& \lambda_{2}^{*} \mathbf{k}_{b_{2}}=1 \otimes S q^{1} \alpha_{3}+1 \otimes S q^{2} S q^{1} \alpha_{1}+w_{3} \otimes \alpha_{1} .
\end{aligned}
$$

The third stage of an MPT is

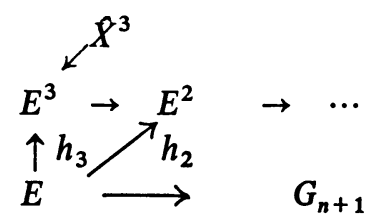

where $\hat{X}^{3}=K_{1}\left(Z_{2}, n+2\right) \times K_{2}\left(Z_{2}, n+3\right)\left(K_{i}=K_{b_{i}}\right)$.

4.4. The space $X^{4}$ is $(n+2)$-connected and $\pi_{n+3}\left(X^{4}\right)=Z_{2}$. The only spherical set we are interested in is $\mathscr{S}^{n+3}\left(X^{4} ; Z_{2}\right)$ which will contain only the characteristic class $c$ of $X^{4}$. Consider the sequence

$$
\left.H^{n+3}\left(X^{4}\right) \stackrel{\hat{\delta}^{*}}{\rightarrow} H^{n+4}\left(\hat{X}^{3}\right) \underset{h_{3}^{*}}{H^{n+4}\left(X^{3}\right)}\right|_{\hat{\delta}_{1}^{*}} ^{H^{n+5}}\left(\hat{X}^{2}\right) .
$$

Now $\hat{\delta}_{1}^{*} h_{3}^{*} \alpha_{b_{1}}=S q^{2} \alpha_{1}$ and $\hat{\delta}_{1}^{*} h_{3} \alpha_{b_{2}}=S q^{1} \alpha_{3}+S q^{2} S q^{1} \alpha_{1}$. Then, since $\hat{\delta}_{1}^{*}$ is a monomorphism, $\operatorname{ker} h_{3}^{*}$ (in dimension $n+4$ ) is generated by $S q^{1} \alpha_{b_{2}}+S q^{2} \alpha_{b_{1}}$. Indeed by direct computation we have

4.4.2 $S q^{1} S q^{1} \alpha_{2}=0$ and $S q^{1}\left(S q^{2} S q^{1}+w_{3} \cdot\right) \alpha_{1}+\left(S q^{2}+w_{2} \cdot\right)\left(S q^{2}+w_{2} \cdot\right) \alpha_{1}=0$. As in the proof of 4.3 .4 we have

LEMMA 4.4.3

$$
S q^{1} \mathbf{k}_{b_{2}}+\left(S q^{2}+w_{2} \cdot\right) \mathbf{k}_{b_{1}}=0
$$

As above, using a sequence analogous to 4.2 .4 we have

$$
\bar{\lambda}_{3}^{*} \mathbf{k}_{c}=1 \otimes S q^{1} \alpha_{b_{2}}+1 \otimes S q^{2} \alpha_{b_{1}}+w_{2} \otimes \alpha_{b_{1}} .
$$

The fourth stage of the MPT is

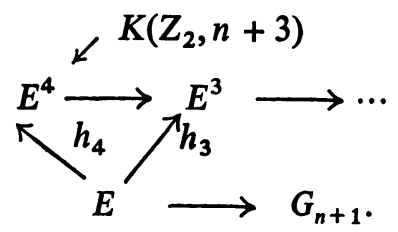




\section{Obstruction theory.}

5.1. Suppose $\mathfrak{b}=(E, B G, p)$ is the universal bundle for a fiber space having $G$ as group and $F$ as fiber. Let $\mathfrak{b}^{\prime}=\left(E^{\prime}, B^{\prime}, p^{\prime}\right)$ be any other $G$-bundle with $F$ as fiber. Suppose $B^{\prime}$ is a $\mathrm{CW}$ complex of cohomology dimension $t$. Consider the diagram

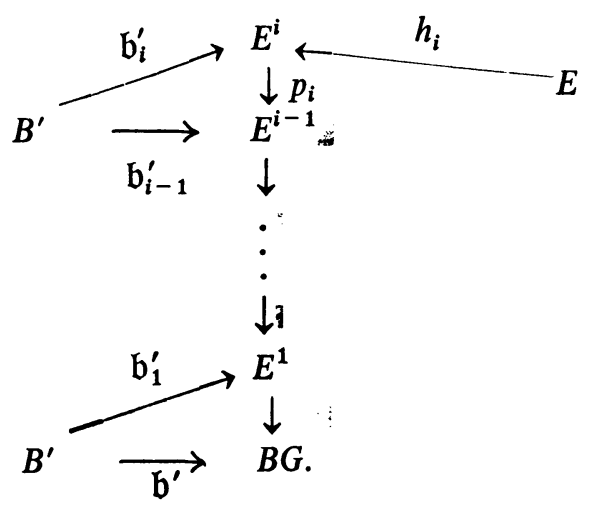

The tower on the right is an MPT of dimension $t$. In particular if $E_{i}$, is the last space in the tower (2.2.5) then the fiber of $h_{i^{\prime}}: E \rightarrow E^{i^{\prime}}$ is $t$-connected. Hence any map $f: B^{\prime} \rightarrow E^{\prime}$ can be lifted to a map $f^{\prime}: B^{\prime} \rightarrow E$.

Recall (2.2) that $\mathfrak{b}^{\prime}$ has a cross section iff $\mathfrak{b}^{\prime}: B^{\prime} \rightarrow B G$ can be lifted to a map $\mathfrak{b}^{\prime}: B^{\prime} \rightarrow E$. Now this is equivalent to lifting $\mathfrak{b}^{\prime}$ to $\mathfrak{b}_{1}^{\prime}, \cdots$, then lifting $\mathfrak{b}_{i-1}^{\prime}$ to $\mathfrak{b}_{i}^{\prime}, \cdots$, until we have $\mathfrak{b}_{i^{\prime}}^{\prime}: B^{\prime} \rightarrow E^{i^{\prime}}$. The last step then is trivial.

This well-known result settles the problem.

THEOREM 5.1.2. Any map $\mathfrak{b}_{i-1}^{\prime}: B^{\prime} \rightarrow E^{i-1}$ can be lifted to a map $\mathfrak{b}_{i}^{\prime}: B^{\prime} \rightarrow E^{i}$ iff $\mathfrak{b}_{i-1}^{\prime *}\left(\mathbf{k}_{a}^{i}\right)=0$ for all $a \in \mathscr{S}_{t_{i}}\left(X^{i}\right)$.

The proof is given in [1, Theorem 3.3.8].

5.2. Now it is clear that we need to know how large a set $\mathfrak{b}_{i-1}^{\prime *}\left(\mathbf{k}_{a}^{i}\right)$ can be if we let $\mathfrak{b}_{i-1}^{\prime *}$ range over all possible liftings. We have investigated this in complete detail for the special case of $\S 4$ but the results seem to be too complicated to be really useful. A much easier question is the following: Suppose $\mathfrak{b}_{i-2}^{\prime}: B^{\prime} \rightarrow E^{i-2}$ is kept fixed. What does $\mathfrak{b}_{i-1}^{\prime *}\left(\mathbf{k}_{a}^{i}\right)$ look like if we consider all possible liftings of $\mathfrak{b}_{i-2}^{\prime}$ ? This question is answered in 5.4.3 but first we prove an interesting result (5.3.3) valid only at the first stage.

5.3. As data for the next theorem consider the sequence

$$
H^{*}\left(E^{\prime}\right) \stackrel{i^{*}}{\rightarrow} H^{*}(F) \stackrel{\delta^{*}}{\rightarrow} H^{*}\left(E^{\prime}, F\right)
$$

LEMMA 5.3.1. The classifying map $\mathfrak{b}^{\prime}$ of $\mathfrak{b}^{\prime}$ can be lifted to a map $\mathfrak{b}_{1}^{\prime}: B^{\prime} \rightarrow E^{1}$ iff $\delta^{*} a=0$ for every $a \in \mathscr{S}_{t_{1}}(F)$. 
Proof. By naturality the tower induced by $\mathfrak{b}^{\prime}$ from a MPT for $\mathfrak{b}$ is a MPT for $\mathfrak{b}^{\prime}$. Hence $\mathfrak{b}^{\prime *}\left(\mathbf{k}_{a}^{1}\right)$ will be the $k$-invariant for a MPT for $\mathfrak{b}^{\prime}$. Lemma 2.2 .8 and Theorem 5.1.2 now complete the proof.

Consider the diagram

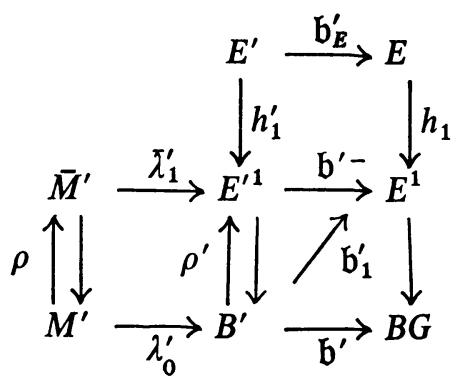

where the lifting $b_{1}^{\prime}$ is chosen so that $b_{1}^{\prime}=b^{\prime-} \rho^{\prime}$. Observe that if $b^{\prime *} \mathbf{k}_{a}^{1}=0$ for all $a$, then $E^{\prime 1}$ is homeomorphic to $B^{\prime} \times \hat{X}^{1}$ and, therefore, if $\rho^{\prime}$ is chosen so that $\bar{\lambda}^{\prime} \rho=\rho^{\prime} \lambda_{0}^{\prime}$, (in this paragraph let $\bar{\lambda}^{\prime}=\bar{\lambda}_{1}^{\prime}$ ), then, in dimensions less than $2 n$, $\bar{\lambda}^{\prime *}$ is an isomorphism between $\operatorname{ker} \rho^{\prime *}$ and $\operatorname{ker} \rho^{*}$. Let $\pi$ be the inverse map, i.e., $\pi: \operatorname{ker} \rho^{*} \rightarrow \operatorname{ker} \rho^{*}$ defined only in dimensions less than $2 n$.

THEOREM 5.3.3. If $B^{\prime}$ is a $\mathrm{CW}$ complex, then

$$
p^{\prime *} \mathfrak{b}_{1}^{\prime *} \mathbf{k}_{a}^{2}=-h_{1}^{\prime *} \pi \bar{\lambda}_{1}^{\prime *} \mathfrak{b}^{\prime-*} \mathbf{k}_{a}^{2} \text {. }
$$

Before we prove this formula, we will illustrate what it says. Suppose $\mathfrak{b}^{\prime}=\left(E^{\prime}, B^{\prime}, p^{\prime}\right)$ is an $n$-sphere bundle over $B^{\prime}$ with zero Euler class. Then using 4.1.1 we have

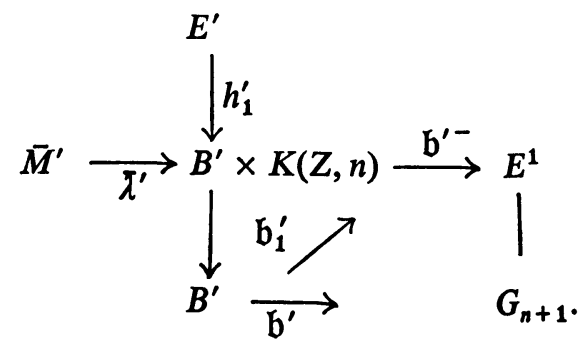

Let $a$ be $h_{1}^{\prime *} \alpha$. Now $\bar{\lambda}^{\prime} \mathbf{k}_{a_{1}}^{2}=w_{2}\left(\mathfrak{b}^{\prime}\right) \otimes \alpha+1 \otimes S q^{2} \alpha$ and therefore $h_{1}^{\prime *} \pi \bar{\lambda}^{\prime *} \mathbf{k}_{a_{1}}^{2}=w_{2}\left(\mathfrak{b}^{\prime}\right) \cdot a+S q^{2} a$. That this equals $p^{\prime *} \mathfrak{b}_{1}^{\prime *} \mathbf{k}_{a_{1}}^{2}$, which is just the classical second obstruction, is Liao's theorem [9].

Proof of the theorem. Refer to diagram 5.3.2. The cohomology of $E^{\prime 1}$ is isomorphic to $\operatorname{ker} \rho^{\prime *} \otimes \operatorname{im} p_{1}^{\prime *}$. Clearly $\pi \bar{\lambda}^{\prime *}\left(\mathfrak{b}^{\prime-}\right)^{*} \mathbf{k}_{a}^{2} \in \operatorname{ker} \rho^{\prime *}$ and hence there is a $z \in H^{*}\left(B^{\prime}\right)$ such that $p_{1}^{\prime *} z=\pi \bar{\lambda}^{\prime *}\left(\mathfrak{b}^{\prime-}\right)^{*} \mathbf{k}_{a}^{2}-\left(\mathfrak{b}^{\prime-}\right)^{*} \mathbf{k}_{a}^{2}$. But $z=\rho^{\prime *} p_{1}^{\prime *} z=-\rho^{\prime *}\left(\mathfrak{b}^{\prime-}\right)^{*} \mathbf{k}_{a}^{2}$ $=-\mathfrak{b}_{1}^{\prime *} \mathbf{k}_{a}^{2}$. Since $h_{1}^{\prime *}\left(\mathfrak{b}^{\prime-}\right)^{*} \mathbf{k}_{a}^{2}=0$ and $h_{1}^{\prime *} p_{1}^{\prime *}=p^{\prime *},-p^{\prime *} \mathfrak{b}_{1}^{\prime *} \mathbf{k}_{a}^{2}=h_{1}^{\prime *} \pi \bar{\lambda}^{\prime *}\left(\mathfrak{b}^{\prime-}\right)^{*} \mathbf{k}_{a}^{2}$.

5.4. Suppose now that we have a lifting of $\mathfrak{b}^{\prime}$ to $\mathfrak{b}_{i-2}^{\prime}: B^{\prime} \rightarrow E^{i-2}$. 
DefinITION 5.4.1. For each $a \in \mathscr{S}_{t_{i-1}}\left(X^{\mathfrak{i}-1}\right)$ let $\mathbf{k}_{a}^{i}\left(\mathfrak{b}^{\prime}\right)=\left\{z \in H^{*}\left(B^{\prime}\right)\right.$; there exists a lifting of $\mathfrak{b}_{i-2}^{\prime}, \mathfrak{b}_{i-1}^{\prime}$ such that $\left.\mathfrak{b}_{i-1}^{\prime *} \mathbf{k}_{a}^{i}=z\right\}$.

The $\operatorname{set}_{a}^{i}\left(b^{\prime}\right)$ will be a coset of certain groups which we now will describe.

Definition 5.4.2.(Compare 3.3.1.) Let $\mathrm{z}=\left(z_{a(v, k)}, \in H^{k}\left(B^{\prime} ; Z\right)\right.$ for each $\left.a \in \mathscr{S}_{t}\left(X^{i-1}\right)\right)$ and let $m_{j-\mu} \otimes x_{\mu} \in H^{\prime}\left(\bar{M} ; Z_{p}\right) \cap \operatorname{ker} \rho^{*}, j<2 n$. Then by $\left(m_{j-\mu} \otimes x_{\mu}\right)^{\prime}(\mathbf{z})$ we mean $\left(\mathfrak{b}_{i-2}^{\prime *} \lambda_{i-2}^{*-1} m_{j-\mu}\right) \cdot \Sigma_{a \in \mathscr{S}_{t}}\left(X^{i-1}\right) \phi_{a} z_{a}$. By linearity this defines $\sigma^{\prime}(\mathbf{z})$.

Let $\bar{\lambda}_{i-1}^{*} \mathbf{k}_{a}^{i}=\sigma_{a} \in H^{*}\left(\bar{M}_{i-1}\right)$.

THEOREM 5.4.3. In the above notation $\mathbf{k}_{a}^{i}\left(b^{\prime}\right)$ is a coset of the group $\sigma_{a}^{\prime}(\mathbf{z})$ where $z_{a(v, k)}$ ranges over all of $H^{k}\left(B^{\prime} ; Z_{v}\right)$.

Before we prove the theorem we will illustrate what it says. Suppose $\mathfrak{b}^{\prime}=\left(E^{\prime}, B^{\prime}, p^{\prime}\right)$ is an $n$-sphere bundle over $B^{\prime}$ with zero Euler class. Now $\sigma_{a_{1}}=w_{2} \otimes \alpha+1 \otimes S q^{2} \alpha$. Let $z$ be a one-tuple $z \in H^{n}\left(B^{\prime} ; Z\right)$. Then $\sigma_{a_{1}}^{\prime}(z)=w_{2}\left(\mathfrak{b}^{\prime}\right) \cdot z$ $+S q^{2} z$ and $\mathbf{k}_{a_{1}}\left(\mathfrak{b}^{\prime}\right)$ is a coset of the group $\left(S q^{2}+w_{2}\right) H^{n}\left(B^{\prime} ; Z\right)$.

Proof of the theorem. Consider the diagram

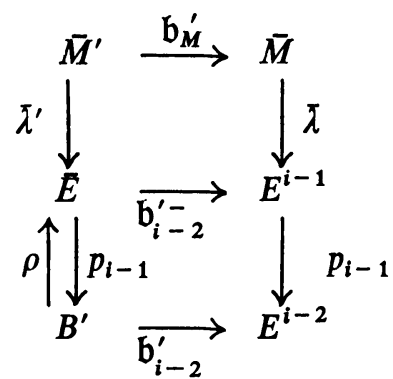

where $\bar{\lambda}=\bar{\lambda}_{i-1}$ and the subscripts on $\bar{M}_{i-1}$ have been suppressed. We can choose representations of $\bar{M}, \bar{E}$, and $\bar{M}$ as products so that

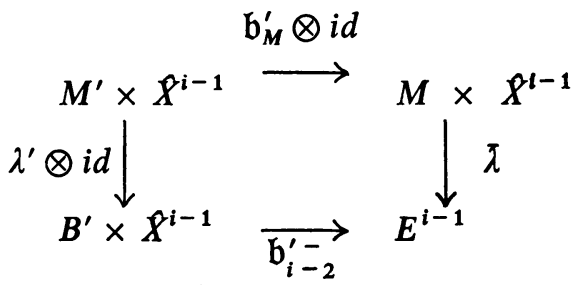

is commutative where each map from the upper left corner acts on each factor separately. In this representation $\rho^{\prime}$ is the usual embedding in the first coordinate. Let $\gamma: B^{\prime} \rightarrow \hat{X}^{i-1}$ be arbitrary. Then $\bar{\rho}^{\prime}=(i d, \gamma): B^{\prime} \rightarrow E$ is another cross section. In dimensions less than $2 n, \lambda^{\prime *}$ is a monomorphism on $\operatorname{ker} \rho^{\prime *}$. Let $\pi$ be the inverse mapping, that is, $\pi: \bar{\lambda}^{\prime *}\left(\operatorname{ker} \rho^{\prime *}\right) \rightarrow \operatorname{ker} \rho^{\prime *}$. Let $\mathbf{z}=\left(z_{a}\right)$ where $z_{a}=\gamma^{*} \alpha_{a}$. Then if $\sigma \in H^{*}(\bar{M}) \cap \operatorname{ker} \rho^{*}$ (Definition 5.4.2), then $\sigma^{\prime}(\mathbf{z})=\bar{\rho}^{\prime *} \pi \mathfrak{b}_{M}^{\prime *} \sigma$. Now 
$\mathfrak{b}_{i-2}^{\prime-*} \mathbf{k}_{a}^{i}-p_{i-1}^{*} \rho^{\prime *} \mathfrak{b}_{i-2}^{\prime-*} \mathbf{k}_{a}^{i} \in \operatorname{ker} \rho^{\prime *}$ and hence it must equal $\pi \mathfrak{b}_{M}^{\prime *} \lambda^{*} \mathbf{k}_{a}^{i}$. In other words $\mathfrak{b}_{i-2}^{\prime-} \mathbf{k}_{a}^{i}=p_{i-1}^{*} \rho^{\prime *} \mathfrak{b}_{i-2}^{\prime-*} \mathbf{k}_{a}^{i}+\pi \mathfrak{b}_{M}^{\prime *} \lambda^{*} \mathbf{k}_{a}^{i}$. Then $\bar{\rho}^{*} \mathfrak{b}_{i-2}^{\prime}{ }^{*} \mathfrak{b}_{a}^{i}=\rho^{\prime *} \mathbf{k}_{i-2}^{\prime-*} \mathbf{k}_{a}+\bar{\rho}^{*} \pi \mathfrak{b}_{M}^{\prime *} \lambda{ }^{*} \mathbf{k}_{a}^{i}$. Letting $\mathfrak{b}_{i-1}^{\prime}=\mathfrak{b}_{i-2}^{\prime-} \rho^{\prime}$ and $\mathfrak{b}_{i-1}^{\prime}=\mathfrak{b}_{i-2}^{\prime-} \bar{\rho}$ we have $\left(\mathfrak{b}_{i-1}^{\prime *}-\mathfrak{b}_{i-1}^{\prime *}\right)\left(\mathbf{k}_{a}^{i}\right)=\sigma^{\prime}(z)$. Since any lifting of $b_{i-2}$ can be compared to $b_{i-1}^{\prime}$ as was done for $\bar{b}_{i-1}^{\prime}$, the theorem now follows from the standard result on classifying maps $\gamma: B^{\prime} \rightarrow X^{i-1}$.

6. Orientable $V_{n+m, m}$ bundles. In this section we will construct the tower for orientable $V_{n+m, m}$ bundles. Let $\mathfrak{g}^{m}=\left(E, G_{n+m}, p\right)$ be the universal orientable $V_{n+m, m}$ bundle. It is well known that $E$ is homotopically equivalent to $G_{n}$ and $M$ can be taken as $G_{n}$ with $\lambda: G_{n} \rightarrow G_{n+m}$ being the usual embedding.

6.1. Our first task will be to compute $\mathscr{S}^{k}\left(V_{n+m, m} ; Z_{q}\right)$ for $q=0$ or 2 . No other primes occur since $H^{*}\left(V_{n+m, m}\right)$ has no odd torsion [3]. We will use the following fibering

$$
V_{n+s, s} \stackrel{i_{s}}{\longrightarrow} V_{n+m, m} \stackrel{\mathbf{p}_{s}}{\longrightarrow} V_{n+m, m-s} .
$$

From Borel [3] we recall that if $n+m<2 n$ then

$$
\begin{aligned}
H^{n}\left(V_{n+m, m} ; Z\right) & =Z & & \text { if } m \equiv 1 \text { or } n=0 \bmod 2, \\
& =0 & & \text { otherwise. } \\
H^{i}\left(V_{n+m, m} ; Z\right) & =0 & & \text { if } n<i<n+m-1, i \equiv 1 \bmod 2 \\
& =Z_{2} & & \text { if } n<i<n+m-1, i \equiv 0 \bmod 2 \\
H^{n+m-1}\left(V_{n+m, m} ; Z\right) & =Z_{2} & & \text { if } n+m \equiv 0 \bmod 2 \\
& =Z & & \text { if } n+m \equiv 1 \bmod 2 .
\end{aligned}
$$

Choose generators of the groups $a_{i} \in H^{n+i}\left(V_{n+m, m} ; J_{i}\right)$ where $J_{i}$ is $Z_{2}$ unless $i=0$ and either $m=1, n=0 \bmod 2$, or $i=m-1$ when $J_{i}=Z$.

LeMma 6.1.3. If $\mathbf{p}_{i^{*}}: \pi_{n+i}\left(V_{n+m, m}\right) \rightarrow \pi_{n+i}\left(V_{n+m, m-i}\right)$ is an epimorphism, then $a_{i}$ satisfies 2.1.1.3.

Proof. Let $f: S^{n+i} \rightarrow V_{n+m, m}$ be a class such that $\mathbf{p}_{i} f$ generates $\pi_{n+i}\left(V_{n+m, m-i}\right)$. Since $V_{n+m, m-i}$ is $(n+i-1)$-connected there is a class $\hat{x}$ such that $\left(\mathbf{p}_{i} f\right)^{*} \hat{x}$ generates $H^{n+i}\left(S^{n+i}, J_{i}\right)$. Hence $\mathbf{p}_{i}^{*} \hat{x} \neq 0$ and satisfies 2.1.1.3. Since $\mathbf{p}_{i}^{*} \hat{x}$ must generate $H^{n+i}\left(V_{n+m, m}\right)$, it can be taken as $a_{i}$.

Since $H^{n+i}\left(V_{n+m, m}\right)$ is singly generated, only 2.1.1.3 is nontrivial in defining regular spherical sets. The above lemma and Paechter's computations [15] give

$$
\begin{aligned}
& \text { if } i=0 ; \\
& i=1 \text { and } n=0 \bmod 2 ; \\
& i=2 \text { and } n=1 \bmod 4 ; \text { or } \\
& i=3 \text { and } n=0 \bmod 4 .
\end{aligned}
$$

The group $J_{i}=Z$ if $i=m-1$ or $i=0$ and $n=0 \bmod 2$. Otherwise $J_{i}=Z_{2}$. 
The Steenrod square operations, as computed by Borel in [3], show that all other regular spherical sets are zero.

LEMMA 6.1.5. If $a_{i} \in \mathscr{S}^{n+i}\left(V_{n+m, m} ; Z_{2}\right)$ or if $a_{0} \in \mathscr{S}^{n}\left(V_{n+m, m} ; Z\right)$, then $\mathbf{k}_{a_{i}}^{\mathbf{1}}=w_{n+i+1}$. If $a_{i} \in \mathscr{S}^{n+m-1}\left(V_{n+m, m} ; Z\right)$, then $\mathbf{k}_{a_{i}}^{1}=\chi$.

Proof. Let $n+m=n^{\prime}$. Let $\left(G_{k}, G_{n^{\prime}}, p\right)$ be the universal $V_{n^{\prime}, n^{\prime}-k}$ bundle. The map $\mathbf{p}_{k-n}: V_{n^{\prime}, m} \rightarrow V_{n^{\prime}, n^{\prime}-k}$ induces natural maps

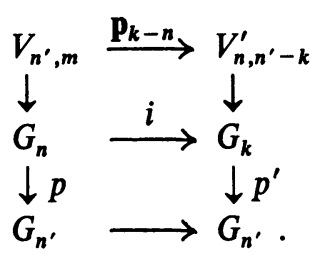

Consider the sequences

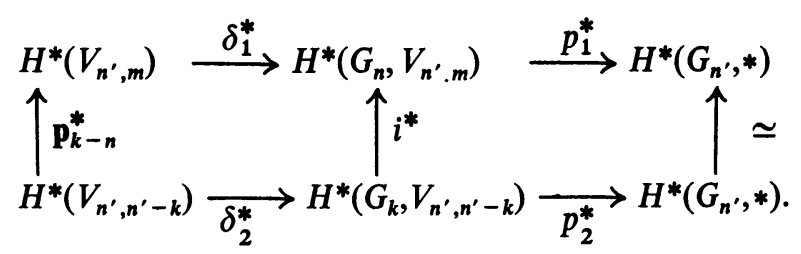

Now the fundamental class $\hat{x}$ of $V_{n^{\prime}, n^{\prime}-k}$ satisfies

$$
\begin{aligned}
\delta_{2}^{*} \hat{x} & =p_{2}^{*} w_{k+1}, & & k \neq n^{\prime}-1, \\
& =p_{2}^{*}( \pm \chi), & & k=n^{\prime}-1 .
\end{aligned}
$$

This together with 2.2.8, 6.1.4, and the commutativity of 6.1 .7 yields the lemma. We can suppose $\chi$ and $a_{m-1}$ are chosen so that the sign ambiguity is decided and $\delta_{2}^{*} \hat{x}=p_{2}^{*} \chi$.

Rather than indexing the $k$-invariants by $\mathscr{S}_{t}\left(V_{n+m, m}\right)$, we use the following conventions:

$$
\begin{aligned}
& \mathbf{k}_{j}^{1}=w_{n+j}, \quad j<m \quad \text { and } j=1 ; \text { or } \\
& j=2 \text { and } n=0 \bmod 2 ; \text { or } \\
& j=3 \text { and } n=1 \bmod 4 \text {; or } \\
& j=4 \text { and } n=0 \bmod 4 ; \text { or } \\
& \mathbf{k}_{m}^{1}=\chi \text { if } m=1, n=1 \bmod 2, \\
&=2, n=0 \bmod 2, \\
&=3, n=1 \bmod 4, \\
&=4, n=0 \bmod 4,
\end{aligned}
$$

and zero otherwise. 
It will make things much easier at a later stage if we add

$$
\begin{aligned}
\mathbf{k}_{5}^{1} & =w_{n+5}, & & n=3 \bmod 8, m>5, \\
& =\chi, & & n=3 \bmod 8, m=5, \\
& =0, & & \text { all other } m \text { and } n .
\end{aligned}
$$

Let $n_{j}=n+j-1$ if $\mathbf{k}_{j}^{1} \neq 0$ and -1 otherwise. The group $J_{j}=Z$ if $m=j$ or $n=0 \bmod 2$ and $j=1$. Otherwise $J_{j}=Z_{2}$. Let $K(\pi,-1)$ be a single point.

Then the first stage of the MPT for $g$ of dimension $t=n+5$ is

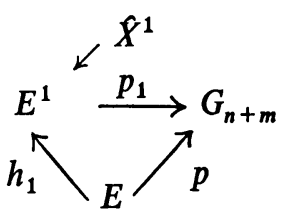

where $\hat{X}^{1}=\prod_{j=1}^{5} K_{j}\left(J_{j}, n_{j}\right)$. Let $\alpha_{j}$ be the image of the characteristic class of $K_{j}$ in $H^{*}\left(\hat{X}^{1}\right)$. When the dependence of $E^{1}$ on $n$ and $m$ is important we will write $E_{n, m}^{1}$. If $t$ is less than $n+5$ we just include as many factors in $\hat{X}^{1}$ for which $n_{j}<t$.

6.2. Consider the sequence

$$
\rightarrow \pi_{*}\left(X^{2}\right) \stackrel{i}{\rightarrow} \pi_{*}\left(V_{n+m, m}\right) \stackrel{h_{*}}{\rightarrow} \pi_{*}\left(\hat{X}^{1}\right) \stackrel{\partial_{*}}{\rightarrow} \pi_{*}\left(X^{2}\right) \rightarrow \cdots
$$

By Paechter's groups we note that $\pi_{k}\left(X^{2}\right)$ is finite for $k \leqq n+4$ except if $n=3 \bmod 4$ and $m=3$ or $n=2 \bmod 4$ and $m=4$. In these cases $\pi_{k}\left(X^{2}\right)$ has rank 1 .

We will look at the case $n=3 \bmod 4$ and $m=3$, the other case being very similar. Consider the sequence with $Z$ for coefficients:

$$
H^{n+2}\left(\hat{X}^{1}\right) \stackrel{h^{*}}{\rightarrow} H^{n+2}\left(V_{n+3,3}\right) \stackrel{i^{*}}{\rightarrow} H^{n+2}\left(X^{2}\right) \stackrel{\hat{\delta}^{*}}{\rightarrow} H^{n+3}\left(\hat{X}^{1}\right)
$$

Since everything in $H^{n+2}\left(\hat{X}^{1}\right)$ is of finite order, the infinite part of $H^{n+2}\left(V_{n+3,3}\right)$, the part generated by $a_{2}$, maps monomorphically into $H^{n+2}\left(X^{2}\right)$. On the other hand, since $S q^{2} a_{0}=\left(a_{2}\right) \bmod 2$, we have $i^{*}\left(S q^{2} \alpha_{1}\right)=\left(a_{2}\right)_{\bmod 2}$. Therefore $h^{*} a_{2}$ is divisible by 2 and $\delta^{*}\left(\frac{1}{2} h^{*} a_{2}\right)=\beta_{2} S q^{2} \alpha_{1}$. Let $f: S^{n+2} \rightarrow V_{n+3,3}$ be a generator of the infinite part. Then $p_{2} f: S^{n+2} \rightarrow S^{n+2}$ is of degree 2 by [15] and hence $f^{*}\left(a_{2}\right)$ is twice a generator of $H^{n+2}\left(S^{n+2} ; Z\right)$. Since $\pi_{n+2}\left(\hat{X}^{1}\right)=0$, there is a map $f: S^{n+2} \rightarrow X^{2}$ such that $f=h f$. Therefore $f^{*}\left(\frac{1}{2} h^{*} a_{2}\right)$ generates $H^{n+2}\left(S^{n+2}\right)$.

By a similar argument we get the other part of

$$
\begin{array}{ll}
\mathscr{S}^{n+m-1}\left(X^{2} ; Z\right)=\left\{\frac{1}{2} h^{*} a_{m-1}\right\} & n=3 \bmod 4, m=3, \\
& n=2 \bmod 4, m=4
\end{array}
$$


Consider the sequences

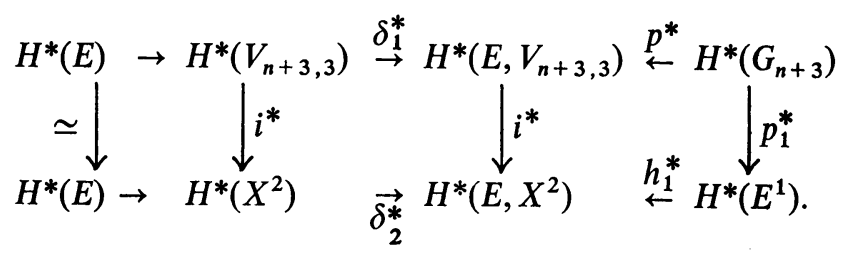

Now $\delta_{1}^{*} a_{2}=p^{*} \chi$ and therefore $2 \delta^{*}\left(\frac{1}{2} h^{*} a_{2}\right)=h_{1}^{*} p_{1}^{*} \chi$. Hence the $k$-invariant belonging to $a_{2} \in \mathscr{S}^{n+2}\left(X^{2} ; Z\right)$ is $\frac{1}{2}\left(p_{1}^{*} \chi\right)$. This is $\mathbf{k}_{5}^{2}$ in Table 6.2.7.

Consider the sequences

$$
\begin{aligned}
6.2 .5 H^{*}\left(E^{1}, \bar{M}_{1}\right) \rightarrow H^{*}\left(E^{1}\right) \stackrel{\lambda_{1}^{*}}{\rightarrow} & H^{*}\left(\bar{M}_{1}\right) \stackrel{\delta^{*}}{\rightarrow} H^{*}\left(E^{1}, \bar{M}_{1}\right) \stackrel{p_{1}^{*}}{\leftarrow} H^{*}\left(G_{n+3,3}, M\right) \stackrel{j^{*}}{\rightarrow} H^{*}\left(G_{n+3}\right) \\
& H^{*} \downarrow \\
& H^{*}\left(\hat{X}^{1}\right) .
\end{aligned}
$$

Then $i^{*} \bar{\lambda}_{1}^{*} \mathbf{k}_{5}^{2}=\beta_{2} S q^{2} \alpha_{1}\left(\beta_{2} S q^{2} \alpha_{2}\right.$ if $\left.n=2 \bmod 4\right)$. Let $\sigma=\beta_{2}\left(w_{2} \otimes \alpha_{1}+1 \otimes S q^{2} \alpha_{1}\right)$. Then $\sigma\left(\mathbf{k}^{1}\right)=0, \sigma \in \operatorname{ker} \rho^{*}$, and $i^{*} \sigma=\beta_{2} S q^{2} \alpha_{1}$. Therefore $\lambda_{1}^{*} \mathbf{k}_{5}^{2}=\sigma$. Theorem 5.4.3 now gives the indeterminacy.

We will use 3.4 .2 to find the $\bmod 2$ and $\bmod 3 k$-invariants. In both these cases $h_{1}^{*}$ is an epimorphism and $\lambda_{1}^{*}$ is a monomorphism. The following theorem, due to $\mathrm{Wu}[17]$ (see also [14]), gives the relations which we need.

THEOREM 6.2.6 [WU]. In $H^{*}\left(G_{n+m} ; Z_{2}\right)$ we have the following relations:

(a) $\left(S q^{2}+w_{2} \cdot\right) w_{n+1}=\xi_{1}(n) w_{n+3}$;

(b) $\left(S q^{4}+w_{4} \cdot\right) w_{n+1}=\xi_{2}(n) w_{n+5}+\left(1+\xi_{1}(n)\right) w_{2} \cdot w_{n+3}+[n+1] w_{3} \cdot w_{n+2}$; where $\xi_{1}(n)=\left(\begin{array}{c}n \\ 2\end{array}\right) \bmod 2, \xi_{2}(n)=\left(\begin{array}{c}n \\ 4\end{array}\right) \bmod 2$ and $[n+1]=(n+1) \bmod 2$. In $H^{*}\left(G_{n+m} ; Z_{3}\right)$ we have the following relation:

(c) If $m=1$ and $n=1 \bmod 2$, then $\mathscr{P}_{3}^{1}(\chi)_{3}+\left(p_{1} \cdot \chi\right)_{3}=0$.

For example if $m \geqq 3$ and $n=2 \bmod 4$, relation (a) becomes $\left(S q^{2}+w_{2} \cdot\right) w_{n+1}+S q^{1} w_{n+2}=0$. If we let $\sigma=1 \otimes S q^{2} \alpha_{1}+w_{2} \otimes \alpha_{1}+1 \otimes S q^{1} \alpha_{2}$ then, in the notation of 6.2 .5 and 3.3.1 $\sigma\left(\mathbf{k}^{1}\right)=0$ and $i^{*} \sigma=S q^{2} \alpha_{1}+S q^{1} \alpha_{2}$. Therefore there is a $k \in H^{*}\left(E^{1}\right)$ such that $\lambda^{*} k=\sigma$ and $\{k\}$ satisfies 3.4.2 for this dimension. Hence $k$ is a $k$-invariant for the next stage and appears as $\mathbf{k}_{1}^{2}$ in the following table. All the entries in the table arise in this fashion. They are listed by giving the relation in $H^{*}\left(G_{n+m}\right)$ which produces them in the fashion described above for $\mathbf{k}_{1}^{2}$. The details involve rather lengthy easy computations and will be omitted.

In Table 6.2.7 the indeterminacy, as computed by 5.4.3, is given. For this purpose we let $\sigma_{1}=\left(S q^{2}+w_{2} \cdot\right)$ and $\sigma_{2}=\left(S q^{4}+w_{4} \cdot\right)$. We also let $A_{k}^{p}=\sigma_{1} H^{k}\left(B ; Z_{q}\right), B_{k}^{p}=\sigma_{2} H^{k}\left(B ; Z_{q}\right), q=0$ or 2 , and $C_{k}=S q^{1} H^{k}\left(B ; Z_{2}\right)$. The results for $m=1$ were also obtained in 4.2. 

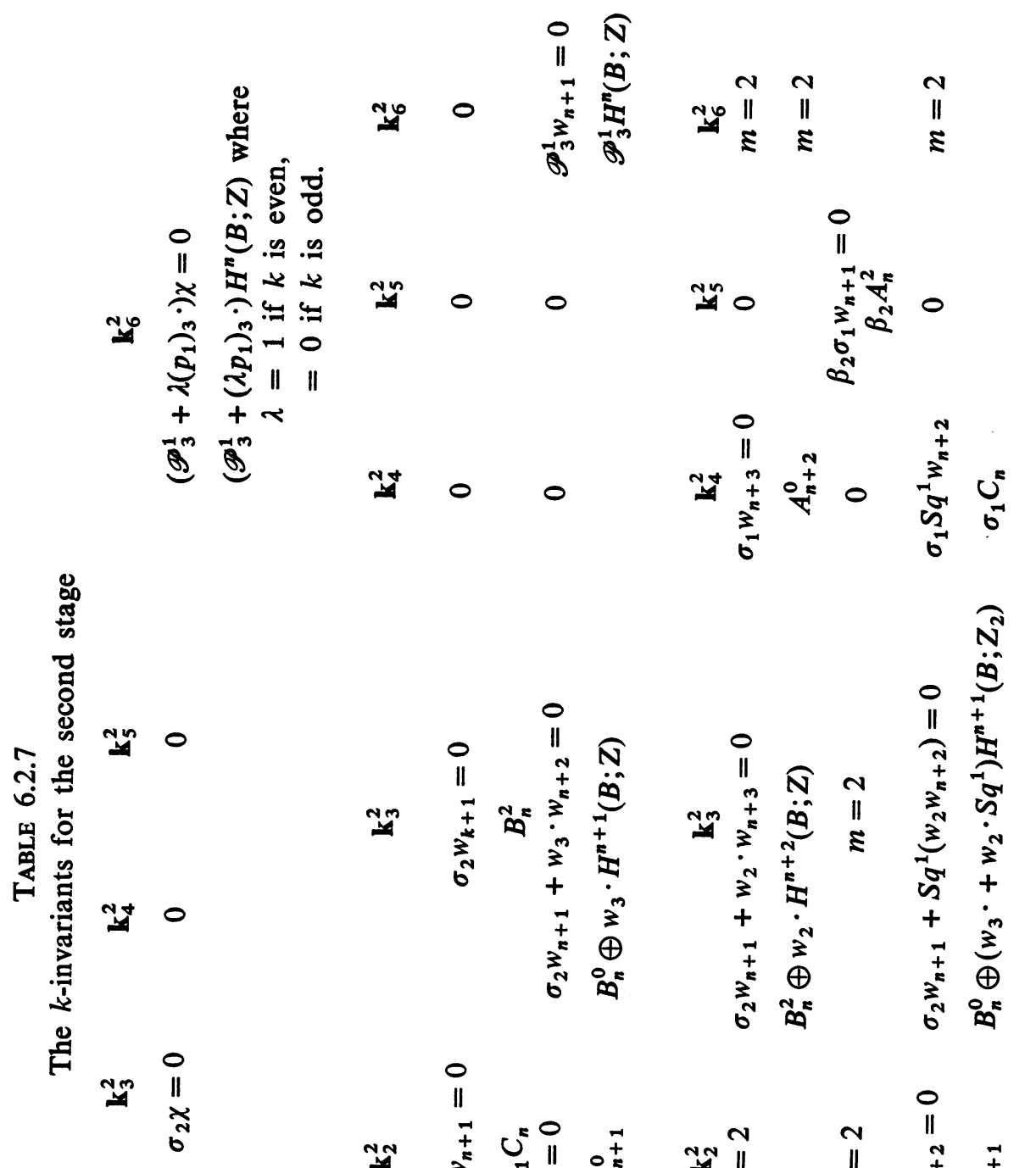

$$
\cong 0
$$

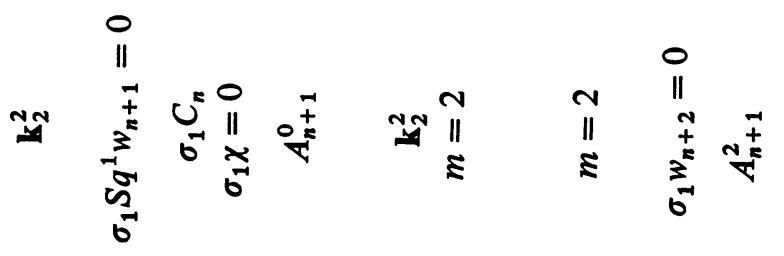$$
\exists \underset{0}{0} \stackrel{0}{x}
$$

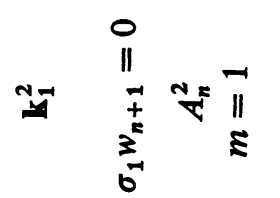

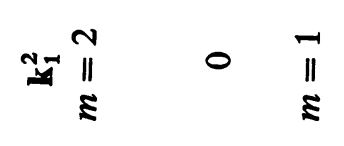

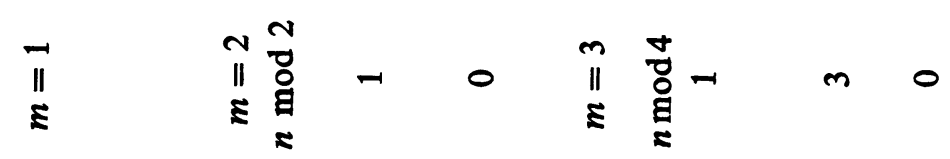


1964] OBSTRUCTION THEORY IN ORIENTABLE FIBER BUNDLES

$\begin{array}{llllll}\pi & j & j & j & j\end{array}$
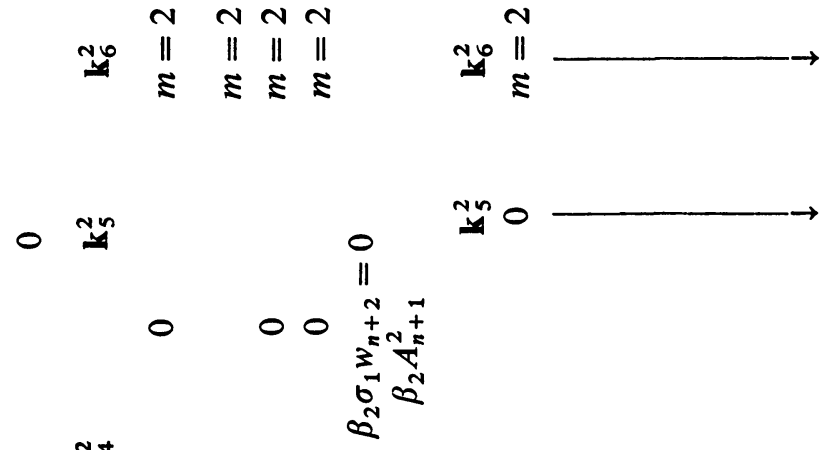

$0 \quad y^{+}$

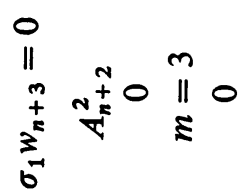<smiles>N#[V][Na]</smiles>

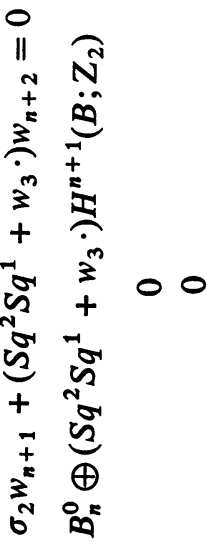

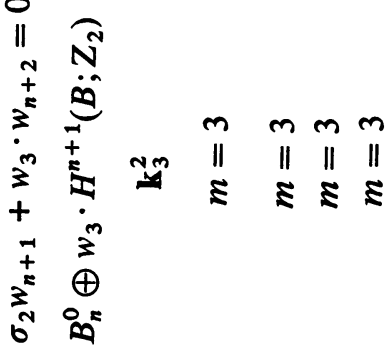<smiles>N#CC(=O)NS(=O)(=O)N[13C](=O)[O-]</smiles>

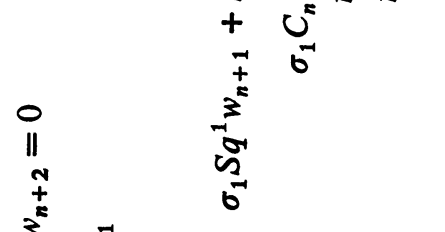

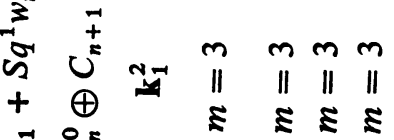

$\stackrel{+}{+}$<smiles>C[SH](C)(=O)[Na]</smiles>

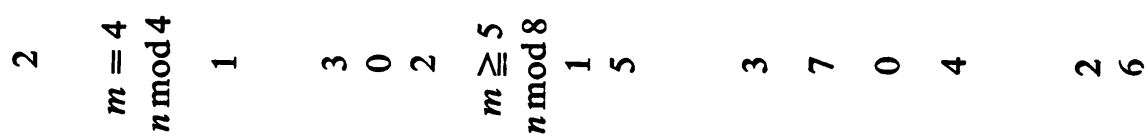


Let $n_{j}=\min (n+j, n+4)$ if $\mathbf{k}_{j}^{2} \neq 0$ and -1 if $\mathbf{k}_{j}^{2}=0$. Let $J_{j}=Z_{2}$ if $j<5 ; J_{5}=Z$ and $J_{6}=Z_{3}$. Then, using the convention that $K(\pi,-1)$ is a point, we have $\hat{X}^{2}=\prod_{j=1}^{6} K_{j}\left(J_{j} ; n_{j}\right)$. The second stage of the MPT for $\mathfrak{b}$ of dimension $n+5$ is

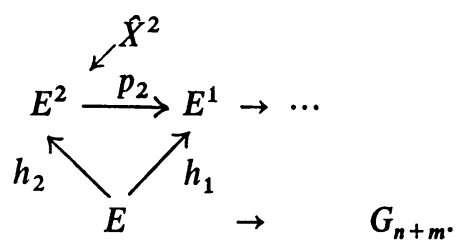

When the dependence of $E^{2}$ on $n$ and $m$ is important we write $E_{n, m}^{2}$. If $t<n+5$ we just consider those factors in $\hat{X}^{2}$ for which $n_{j}<t$.

6.3. Using Paechter's groups [15], we note that $\pi_{*}\left(X_{3}\right)$ has only 2 torsion in dimensions less than $n+5$. Theorem 3.4 .2 will be used to find the new $k$ invariants. All that we need are classes $\sigma \in H^{*}\left(M_{2}\right) \cap \operatorname{ker} \rho^{*}$ such that $\sigma\left(\mathbf{k}^{2}\right)=0$ and $i^{*} \sigma=\sum_{j=1}^{6} \phi_{j} \alpha_{j}$ with at least one $\phi_{j}$ primitive.

The next theorem gives us all the classes which we need. The theorem describes $\sigma$ by the form $\sigma\left(\mathbf{k}^{2}\right)$. The class $\sigma$ is then obtained as in 3.3.1.

THEOREM 6.3.1. The following relations hold in $H^{*}\left(E^{1} ; Z_{2}\right)$.

(a) If $m=1$, or $m=2$ and $n \equiv 0 \bmod 2$, or $n \equiv 0 \bmod 4$,

$$
\sigma_{1} \mathbf{k}_{1}^{2}=0 \text {. }
$$

(b) If $m=2$ and $n \equiv 1 \bmod 2$, or $n \equiv 1 \bmod 4$,

$$
\sigma_{1} \mathbf{k}_{1}^{2}+S q^{1} \mathbf{k}_{2}^{2}=0
$$

(c) If $m=1$ or $n \equiv 0 \bmod 2$,

$$
S q^{1} \mathbf{k}_{3}^{2}+\left(S q^{2} S q^{1}+w_{3} \cdot\right) \mathbf{k}_{1}^{2}=0 .
$$

(d) If $n \equiv 0 \bmod 2, m=3$ or 4 , or $n \equiv 0 \bmod 8, m \geqq 5$,

$$
\sigma_{1} \mathbf{k}_{2}^{2}+S q^{1} \mathbf{k}_{4}^{2}=0
$$

(e) If $n \equiv 4 \bmod 8, m \geqq 5$,

$$
S q^{1} \mathbf{k}_{4}^{2}+\left(S q^{2} S q^{1}+w_{3} \cdot\right) \mathbf{k}_{1}^{2}+\sigma_{1} \mathbf{k}_{2}^{2}=0 .
$$

(f) If $m \equiv 2$, or $n \equiv 1 \bmod 2$ and $m=3$, or $n \equiv 3 \bmod 2$ and $m \geqq 2$,

$$
\sigma_{1} \mathbf{k}_{2}^{2}=0
$$

(g) If $n \equiv 2 \bmod 4$ and $m=3$,

$$
\dot{S} q^{1} \sigma_{1} \mathbf{k}_{1}^{2}+\sigma_{1} \mathbf{k}_{2}^{2}=0
$$


Proof. Consider the diagram

$$
\begin{aligned}
& H^{*}\left(E^{1}, \bar{M}_{1}\right) \stackrel{j_{1}^{*}}{\rightarrow} H^{*}\left(E^{1}\right) \stackrel{\lambda_{1}^{*}}{\rightarrow} H^{*}\left(\bar{M}_{1}\right) \\
& \quad \uparrow \simeq \\
& H^{*}\left(G_{n+m}, M\right) .
\end{aligned}
$$

Using $Z_{2}$ for coefficients, we easily verify $j_{1}^{*}$ is zero in the range of interest. (This is why it was convenient to add $\mathbf{k}_{5}^{1}$ in $6.1 .8^{\prime}$ ). Hence each formula will be verified once it is shown that $\bar{\lambda}_{1}^{*}$ applied to the left side is zero. This is done by direct computation. We will only do (a); the rest work the same way.

$$
\begin{aligned}
\bar{\lambda}_{1}^{*} \sigma_{1} \mathbf{k}_{1}^{2} & =\left(S q^{2}+w_{2} \cdot\right)\left(S q^{2}+w_{2} \cdot\right) \alpha_{1} \\
& =S q^{2} S q^{2} \alpha_{1}+w_{2} \cdot w_{2} \alpha_{1}+w_{2} S q^{2} \alpha_{1}+w_{2} S q^{2} \alpha_{1}+w_{2} w_{2} \alpha_{1} \\
& =S q^{2} S q^{2} \alpha_{1}=0 .
\end{aligned}
$$

The last equation follows since $\alpha_{1}$ is an integer class.

Theorems 6.3.1 and 3.3.4 define a subset $\{k\}$ of $H^{*}\left(E^{2} ; Z_{2}\right)$ which satisfies 1,2 , and 4 of 3.4.2. A check with Paechter's tables, using the remark after 3.4.2, shows that 3 is satisfied also and we have a complete set of $k$-invariants which are given in Table 6.3.3. The arrangement is just as in Table 6.2.7. The relations are identified by reference to 6.3.1. The portion of the table for $m=1$ was done in 4.3. (For Table 6.3.3 see p. 342.)

If $\mathbf{k}_{j}^{3} \neq 0$, let $n_{1}=n+3$ and $n_{j}=n+4$ for $j \geqq 2$. If $\mathbf{k}_{j}^{3}=0$, let $n_{j}=-1$. Then $\hat{X}^{3}=\prod_{j=1}^{3} K_{j}\left(Z_{2}, n_{j}\right)$. The third stage of the tower for $g$ in dimension $n+5$ is

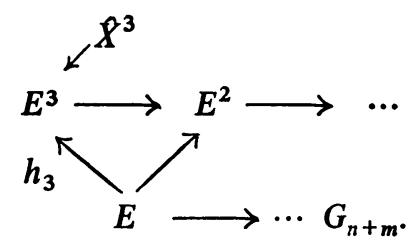

For $t<n+4$ we just take those factors for which $n_{j}<t$.

6.4. The space $X^{4}$ is $(n+3)$-connected and $\pi_{n+4}\left(X^{4}\right)=Z_{2}$ if $m=1$ or if $n=0 \bmod 4$ and is 0 otherwise.

THEOREM 6.4.1. In $H^{*}\left(E^{2} ; Z_{2}\right)$, if $m=1$ or $n \equiv 0 \bmod 4$, we have

$$
S q^{1} \mathbf{k}_{2}^{3}+\sigma_{1}\left(\mathbf{k}_{1}^{3}\right)=0 \text {. }
$$

Proof. Consider the diagram

6.4.2 $H^{*}\left(E^{3}\right) \stackrel{\lambda_{3}^{*}}{\rightarrow} H^{*}\left(\bar{M}_{3}\right) \stackrel{\delta^{*}}{\rightarrow} H^{*}\left(E^{3}, \bar{M}\right) \stackrel{p_{3}^{*}}{\leftarrow} H^{*}\left(E^{2}, n\right) \stackrel{j^{*}}{\rightarrow} H^{*}\left(E^{2}\right) \stackrel{\lambda_{2}^{*}}{\rightarrow} H^{*}\left(\bar{M}_{2}\right)$. 
TABLE 6.3.3;

The $k$-invariants for the third stage

$$
\begin{array}{ccc}
m=1 & \mathbf{k}_{1}^{3} & \mathbf{k}_{2}^{3} \\
& a & c \\
& A_{n+1}^{2} & C_{n+3} \oplus\left(S q^{2} S q^{1}+w_{3} \cdot\right) H^{n+2}\left(B ; Z_{2}\right)
\end{array}
$$$$
\mathbf{k}_{\mathbf{3}}^{\mathbf{3}}
$$$$
0
$$

$$
m=2
$$

$n \equiv 1 \bmod 2$

b

$f$

$$
\begin{aligned}
& A_{n+1}^{2} \oplus C_{n+2} \\
& 0 \\
& A_{n+2}^{2} \\
& 0 \\
& m=1 \\
& m=1 \\
& f \\
& A_{n+2}^{2} \\
& m=3 \\
& \mathbf{k}_{1}^{3} \\
& \mathbf{k}_{2}^{3} \\
& \mathbf{k}_{3}^{3} \\
& n \equiv 1 \bmod 4 \\
& m=2 \\
& m=2 \\
& m=2 \\
& 3 \\
& 0 \\
& m=2 \\
& m=2 \\
& 0 \\
& m=1 \\
& m=2 \\
& A_{n+2}^{2} \oplus C_{n+3} \\
& g \\
& 2 \\
& 0 \\
& m=2 \\
& S q^{1} A_{n+2}^{2} \oplus A_{n+2}^{2} \\
& m=4 \\
& \mathbf{k}_{1}^{3} \\
& \mathbf{k}_{2}^{3} \\
& \mathbf{k}_{3}^{\mathbf{3}} \\
& n \equiv 1 \bmod 4 \\
& m=3 \\
& m=3 \\
& m=3 \\
& \equiv 4 \\
& n \equiv 0 \bmod 8 \\
& \equiv 1 \bmod 2 \\
& \equiv 2 \bmod 4 \\
& m=4 \\
& m=4 \\
& C_{n+3} \oplus\left(S q^{2} S q^{1}+w_{3} \cdot\right) H^{n+1}\left(B ; Z_{2}\right) \oplus A_{n+2}^{2}
\end{aligned}
$$


The new $k$-invariant $\mathbf{k}^{4}$ must satisfy $j^{*} p_{3}^{*}-1 \delta^{*} \lambda_{3}^{*} \mathbf{k}^{4}=0$ and $\lambda_{3}^{*} \mathbf{k}^{4} \in \operatorname{ker} \rho^{*}$ by 3.3.4.

In dimension $n+4 \operatorname{ker} \rho^{*}$ is generated by $1 \otimes S q^{1} \alpha_{2}, 1 \otimes S q^{2} \alpha_{1}, w_{2} \otimes \alpha_{1}$ and $1 \otimes S q^{1} \alpha_{3}$. We see by direct computation using 3.3.3 that only $\sigma=1 \otimes S q^{1} \alpha_{2}+1 \otimes S q^{2} \alpha_{1}+\dot{w}_{2} \otimes \alpha_{1}$ is in the kernel of $\lambda_{2}^{*} j^{*} p_{3}^{*-1} \delta^{*}$ restricted to $\operatorname{ker} \rho^{*}$. Since $j^{*} p_{3}^{*-1} \delta^{*}$ must have a nonzero kernel on $\operatorname{ker} \rho^{*}$, it must be generated by $\sigma$. But $\sigma(\mathbf{k})=S q^{1} \mathbf{k}_{2}^{3}+\sigma_{1} \mathbf{k}_{1}^{3}=j^{*} p^{*-1} \delta^{*} \sigma$ by 3.3.3. This proves the theorem.

Theorem 6.4.1 describes the last $k$-invariant.

6.4.3. $\mathbf{k}_{4} \neq 0$ if $m=1$ or if $n=0 \bmod 4$; it is given by the relation $S q^{1} \mathbf{k}_{2}^{3}+\sigma_{1} \mathbf{k}_{1}^{3}=0$ in the manner of 3.4.2.

The fourth stage of the tower is

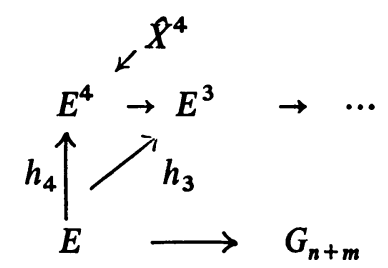

where $\hat{X}^{4}=K\left(Z_{2}, n+3\right)$ if $m=1$ or $n=0 \bmod 4$ and $\hat{X}^{4}=\{*\}$ otherwise.

6.5. There are some useful relations between $k$-invariants, for different values of $m$, for some values of $n$.

6.5.1. Suppose $n \equiv 0 \bmod 4$ and $m>2$ and $t=n+3$. Then $E_{n, m}^{1}(6.1 .9)$ is a fiber space over $G_{n+m}$ having $K_{1}(Z, n) \times K_{2}\left(Z_{2}, n+1\right)$ as fiber and $w_{n+1}$ and $w_{n+2}$ as $k$-invariants respectively. The subset of $E_{n, m}^{1}$ which lies over $G_{n+1}$ is just $E_{n, 1}^{1} \times K_{2}$. There is a natural projection $\pi: E_{n, m}^{1} \rightarrow X$ where $X$ is a fiber space over $G_{n+m}$ having $K_{1}(Z, n)$ as fiber and $w_{n+1}$ as $k$-invariant. Then we have the composite map

$$
\mu: E_{n, 1}^{1} \stackrel{\lambda_{1}}{\subset} E_{n, 1}^{1} \times K_{2}\left(Z_{2}, n\right) \stackrel{\lambda_{2}}{\subset} E_{n, m}^{1} \stackrel{\pi}{\rightarrow} X
$$

which is also an embedding. Let ${ }_{m} \mathbf{k}_{j}^{2} \in H^{*}\left(E_{n, m}^{1}\right)$ be the $k$-invariants. Then we can prove

LEMMA 6.5.3. If $n \equiv 0 \bmod 4$ and $m>2$, then $\lambda_{1}^{*} \lambda_{2}^{*}\left({ }_{m} \mathbf{k}_{1}^{2}\right)={ }_{1} \mathbf{k}_{1}^{2}$.

Proof. Consider the sequence

$$
H^{*}(X) \stackrel{i^{*}}{\rightarrow} H^{*}\left(G_{n} \times K_{1}\right) \stackrel{\delta^{*}}{\rightarrow} H^{*}\left(G_{n+m}, G_{k}\right)
$$

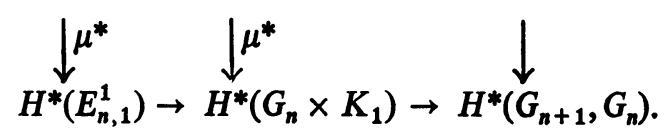


Based on the relation $\left(S q^{2}+w_{2} \cdot\right) w_{k+1}=0$ (following the proof of 3.3.4) there is a class $v_{1}$ in $H^{*}(X)$ (not unique) such that $i^{*} v_{1}=\left(S q^{2}+w_{2} \cdot\right) \alpha_{1}$. Clearly $\mu^{*} v_{1}={ }_{1} \mathbf{k}_{1}^{2}$ and $\pi^{*} v_{1}={ }_{m} \mathbf{k}_{1}^{2}$ hence $\lambda_{1}^{*} \lambda_{2}^{*}\left({ }_{m} \mathbf{k}_{1}^{2}\right)={ }_{1} \mathbf{k}_{1}^{2}$.

Lemma 6.5.5. In $H^{*}(X)$ for $m \geqq 7,\left(S q^{2}+w_{2} \cdot\right) v_{1}=0$.

Proof. From 6.5.4 we see that $\left(S q^{2}+w_{2} \cdot\right) v_{1}$ could only be a linear combination of $w_{n+4}$ and $w_{2} \cdot w_{n+2}$. But $S q^{1}\left(S q^{2}+w_{2}\right)\left(S q^{2}+w_{2}\right)=0$ on all classes and $S q^{1}\left(S q^{2}+w_{2} \cdot\right) w_{2} \cdot w_{n+2}=w_{2} \cdot w_{2} \cdot w_{n+3}$ and $S q^{1}\left(S q^{2}+w_{2} \cdot\right) w_{n+4}=w_{n+7}$. Since there are no polynomial relations among the $w_{i}$, we have the lemma.

As above we have

$$
E_{n, 1}^{2} \stackrel{\lambda_{3}}{\complement} E_{n, m}^{2} \stackrel{\pi_{2}}{\rightarrow} Y
$$

where $Y$ is the fiber space over $X$ with $K\left(Z_{2}, n+1\right)$ as fiber and $v_{1}$ as $k$-invariant. Following the proof of 6.5 .3 we prove easily

Lemma 6.5.7. If $n \equiv 0 \bmod 4, m \geqq 7$, then $\lambda_{3}^{*}\left({ }_{m} \mathbf{k}_{1}^{3}\right)={ }_{1} \mathbf{k}_{1}^{3}$.

If $n \equiv 3 \bmod 4, m \geqq 2$, then there exists $\lambda_{4}: E_{n, 2}^{1} \subset E_{n, m}^{1}$. Indeed in this case $E_{n, 2}^{1}$ is just the subset of $E_{n, m}^{1}$ which lies above $G_{n+2}$. The following lemma is easy.

Lemma 6.5.8. If $n \equiv 3 \bmod 4$ and $m \geqq 2$, then $\lambda_{4}^{*}\left({ }_{m} \mathbf{k}_{2}^{2}\right)={ }_{2} \mathbf{k}_{2}^{2}$.

Remark. This lemma is false for $n \equiv 1 \bmod 4$ and $m \geqq 4$.

\section{Applications.}

7.1. In [7] Hirsch proved that if $M^{n}$ is a differentiable $n$-manifold embedded in $n+k+r(k>0)$ dimensional Euclidean space $\left(M^{n} \subset R^{n k++r}\right)$ with a field of normal $r$-frames then $M^{n}$ immerses in $R^{n+k}\left(M^{n} \subseteq R^{n+k}\right)$. Using this result we prove 7.1.1. In the following $\bar{w}_{i}=w_{i}(\mathfrak{n})$ where $\mathfrak{n}$ is the normal bundle to some embedding of $M^{n}$ in $R^{n+k}$.

THEOREM 7.1.1. Let $M^{n}$ be a compact orientable $n$-manifold $(n>4)$. If $n$ is even, then $M^{n}$ immerses in $R^{2 n-2}$ iff $\bar{w}_{2} \cdot \bar{w}_{n-2}=0$; if $n$ is odd, $M^{n}$ immerses in $R^{2 n-2}$.

Proof. By the Massey-Peterson-Haefliger-Hirsch theorem [12], $M^{n}$ embeds in $R^{2 n-1}$. By a theorem of Massey (Theorem V[11]), $M^{n}$ immerses in $R^{2 n-2}$ if $\bar{w}_{2} \cdot \bar{w}_{n-2}=0$. By the Massey-Peterson theorem [12], $\bar{w}_{n-2}=0$ unless $n=2^{r}$ or $2^{r}+1, r>0$. If $n=1 \bmod 4$, then $M^{n}$ immerses in $R^{2 n-2}$ [7]. So the only case which remains is $n=2^{r}$.

Let $\mathfrak{n}=\left(N, M^{n}, p\right)$ be the normal bundle to an embedding of $M^{n}$ into $R^{2 n-1}$. Let $\mathfrak{n}^{\prime}=\mathfrak{n} \oplus 2 I=\left(N^{\prime}, M^{n}, p\right)$. Then $\mathfrak{n}^{\prime}$ is the stable normal bundle and we will be finished if we show that the second obstruction to a cross section in 
$\mathfrak{n}^{\prime 3}$ (the associate $V_{n+1,3}$ bundle) is $\bar{w}_{2} \cdot \bar{w}_{n-2}$. Let $N_{1}$ and $N_{3}^{\prime}$ be the total space of the associated $n^{1}$ and $\mathfrak{n}^{\prime 3}$ bundles respectively. Then there is a natural map $f$ such that

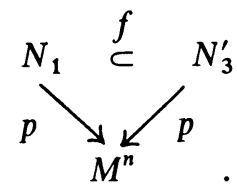

We wish to apply Theorem 5.3.3 to the bundles $\mathfrak{n}^{1}$ and $\mathfrak{n}^{\prime 3}$ and compare the second obstructions. Consider the diagram

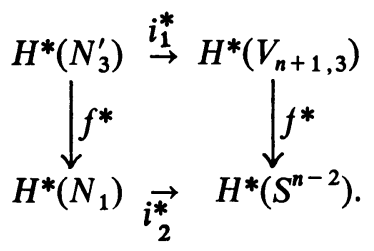

Suppose in the diagram $5.3 .2, \mathfrak{b}$ is the universal $V_{n+1,3}$ tower of $\S 6$ and $\mathfrak{b}^{\prime}$ is the tower for $\mathfrak{n}^{\prime}$. The only possible nonzero obstruction is $\mathbf{k}_{1}^{2}$ (Table 6.2.7). In the notation of 5.3.2, we have

$$
\bar{\lambda}^{*} \mathbf{k}_{1}^{2}=\bar{w}_{2} \otimes \alpha_{1}+1 \otimes S q^{2} \alpha_{1}+1 \otimes S q^{1} \alpha_{2} .
$$

Now $i_{1}^{*}\left(h_{1}^{\prime *} \alpha_{1}\right)$ is the generator of $H^{n-2}\left(V_{n+1,3} ; Z\right)$, where $i_{1}^{*}$ is defined in 7.1.3 and $h_{1}^{\prime}$ is defined in 5.3.2; and $i_{1}^{*}\left(h_{1}^{\prime *} \alpha_{2}\right)$ is nonzero in $H^{n-1}\left(V_{n+1,3} ; Z_{2}\right)$. Hence $f^{*}\left(h_{1}^{\prime *} \alpha_{2}\right)=a$, the class defined in the example after the statement of 5.3.3. Applying 5.3.3, letting $v=h^{*}\left(\mathbf{k}_{1}^{2}\right)$ be the second obstruction class, we have

$$
p^{*} v=\left(S q^{2}+\bar{w}_{2} \cdot\right)\left(h_{1}^{\prime *} \alpha_{1}\right)+S q^{1}\left(h_{1}^{\prime *} \alpha_{2}\right) \text {. }
$$

But $f^{*} p^{*} v=\left(S q^{2}+\bar{w}_{2} \cdot\right) a+S q^{1}\left(f^{*} h_{1}^{\prime *} \alpha_{2}\right)$. By Massey's theorem $\left(S q^{2}+\bar{w}_{2} \cdot\right) a$ $=p^{*}\left(\bar{w}_{2} \cdot \bar{w}_{n-2}\right)$ and therefore $p^{*}\left(v+\bar{w}_{2} \cdot \bar{w}_{n-2}\right) \in S q^{1} H^{n-1}\left(N_{1} ; Z_{2}\right) \cap p^{*}\left(H^{n}\left(M^{n} ; Z_{2}\right)\right)$ $=0$. Since $p^{*}$ is a monomorphism we are finished.

7.2. The following result, due to Epstein and Schwarzenberger, relates the finding of cross sections and the embedding of projective spaces. The result is a slight generalization of a theorem in [4] and we give the proof here with their permission. The symbol $\subset$ will be used for "embeds" throughout the rest of this paper.

THEOREM 7.2.1. If $n H_{q} \subset R^{p}$ (Definition 1.2.5) and $P_{n-1} \subset S^{m-1}$ then $P_{n+q} \subset R^{p+m}$. This can be made differentiable if $2(p+m) \geqq 3(n+q+1)$.

Proof. Let $P_{n-1}$ be the usually embedded subset of $P_{n+q}$. Then $P_{n+q}-P_{n-1}=n H_{q}$. Let $B$ be the $n-1$ sphere bundle associated with $n H_{q}$. Then there exists a map $g: B \rightarrow P_{n-1}$ such that $P_{n+q}=n H_{q} \cup_{g} P_{n-1}$. We can represent points in $P_{n+q}$ 
by pairs $(b, r)$ where $b \in B$ and $r \in[0,1]$. When $r$ is 1 we identify $B$ with $P_{n-1}$ using $g$. Let $h: n H_{q} \subset R^{p}$ and $h_{1}: P_{n-1} \subset S^{m-1} \subset R^{m}$. Then

$$
F(b, r)=\left((1-r) h(b, r), r h_{1}(g(b))\right)
$$

is a 1-1 continuous mapping of $n H_{q} \cup_{g} P_{n-1}$ into $R^{p+m}$. If $2(p+m) \geqq 2(n+q+1)$, then this topological embedding can be approximated by a differentiable one [5].

We apply this result by asking if $n H_{q}$ can be embedded in the normal bundle $\mathfrak{n}$ of an embedding of $P_{q}$ in $R^{p}$. Epstein and Schwarzenberger proved in [4] that $H_{q}$ embeds in $\mathfrak{n}$ iff $\mathfrak{n} \otimes H_{q}$ has a nonzero cross section. If we find $n$-linearly independent cross sections of $\mathfrak{n} \otimes H_{q}$, then the $n$ embeddings of $H_{q}$ produced by the cross sections can be used to give an embedding of $n H_{q}$ in $\mathfrak{n}$. A computation [4, Proposition 4] shows that if $\mathfrak{n}$ is the normal bundle of an embedding of $P_{q}$ in $R^{p}$ then $\mathfrak{n} \otimes H_{q}$ is of the same stable bundle type as $(p+1) H_{q}$. From this formula we can readily compute the StiefelWhitney classes of $\mathfrak{n} \otimes H_{q}$. In particular, $\mathfrak{n} \otimes H_{q}$ is orientable iff $p=1 \bmod 2$. We shall prove the following embedding theorem.

THEOREM 7.2.2. The following embeddings of real projective spaces are possible:

(a) If $k \equiv 3 \bmod 4, k>3$, then $P_{k} \subset R^{2 k-2}$.

(b) If $k \equiv 0 \bmod 2, k \neq 2^{j}$ or $2^{j}+2$, then $P_{k} \subset R^{2 k-3}$.

(c) If $k \equiv 1 \bmod 4, k \neq 2^{j}+1$, then $P_{k} \subset R^{2 k-3}$.

The proof will proceed by showing certain bundles over projective spaces always have a cross section. Some of these are of interest and so we isolate them.

THEOREM 7.2.3. If $k=3 \bmod 4$, then every orientable $k-2$ plane bundle $\mathfrak{b}$ over $P_{k}$ or $P_{k-1}$ has a nonzero cross section.

Proof. If $k=3$, the theorem is trivial. So suppose $k>3$. The Euler class is an integer class in $H^{k-2}\left(P_{k} ; Z\right)=0$ and therefore is zero. If $w_{2}(\mathfrak{b}) \neq 0$, then the indeterminacy of $\mathbf{k}_{1}^{2}$ is all of $H^{k-1}\left(P_{k} ; Z_{2}\right)$. Therefore the classifying map can be lifted to $E_{k-3,1}^{2}$. Again the indeterminacy of $\mathbf{k}_{1}^{3}$ is all of $H^{k}\left(P_{k} ; Z_{2}\right)$ and this proves that a cross section exists.

Suppose $w_{2}(\mathfrak{b})=0$. By an unpublished theorem of Atiyah and Hirzebruch there exists an integer $m \geqq 7$ and an $k+m-2$ plane bundle over $\boldsymbol{P}_{\boldsymbol{k}+5}, \mathfrak{a}$, such that $\mathfrak{b} \otimes(m-1) I=i^{-1} \mathfrak{a}$ (the bundle induced by $i$ ) where $i: P_{k} \subset P_{k+5}$. Let $n=k-3$ and construct the tower 6.1 .9 and 6.2 .8 for $V_{n+m, m}$ bundles and for $V_{n+1,1}$ bundles. Since we are only interested in obstructions up to dimension $n+2$, we modify the tower so that it is a MPT of dimension $n+2$. Then there exist liftings $\mathfrak{b}_{1}$ and $\mathfrak{a}_{1}$ of the classifying maps of $\mathfrak{b}$ and $\mathfrak{a}$ such that the lower portion of the diagram 


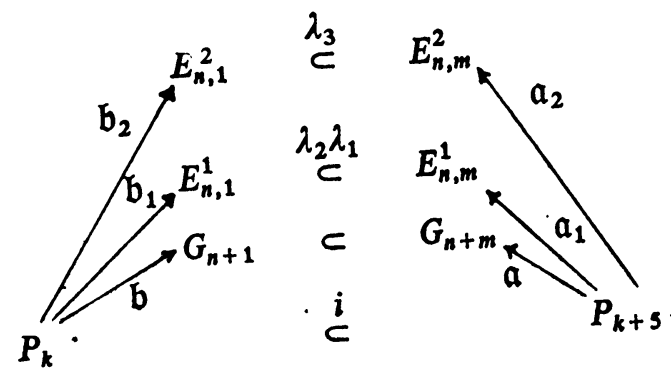

is commutative (at least up to homotopy). By 6.3.1(a), $S q^{2} \mathfrak{a}_{1}^{*}\left({ }_{m} \mathbf{k}_{1}^{2}\right)=0$ but $S q^{2}$, acting on $H^{k-1}\left(P_{k+5} ; Z_{2}\right)$, is an isomorphism. Hence $\mathfrak{a}_{1}^{*}\left({ }_{m} \mathbf{k}_{1}^{2}\right)=0$ and 6.5.3 implies $\mathfrak{b}_{1}^{*} \mathbf{k}_{1}^{2}=0$. Hence $\mathfrak{b}_{2}$ and $\mathfrak{a}_{2}$ can be defined. Again the values of $\mathfrak{a}_{2}^{*}\left({ }_{m} \mathbf{k}_{1}^{3}\right)$ and $b_{2}^{*}\left(\mathbf{k}_{1}^{3}\right)$ are independent of the choice of $\mathfrak{a}_{2}$ and $\mathfrak{b}_{2}$, respectively. Using 6.4.1, we see that $S q^{1} \sigma_{1}\left({ }_{m} \mathbf{k}_{1}^{3}\right)=0$ in $H^{*}\left(E_{n, m}^{2}\right)$. Hence $S q^{3} \mathfrak{a}_{2}^{*}\left({ }_{m} \mathbf{k}_{1}^{3}\right)=0$; but, in this dimension, $S q^{3}$ is also an isomorphism. Hence $\mathfrak{a}_{2}^{*}\left({ }_{m} \mathbf{k}_{1}^{3}\right)=0$ and, by 6.5.7, we see that $\mathfrak{a}_{2}^{*}\left({ }_{m} \mathbf{k}_{1}^{3}\right)=\mathfrak{b}_{2}^{*}\left(\mathbf{k}_{1}^{3}\right)=0$ and this proves 7.2.3.

We now prove 7.2.2(a). Theorem 7.2.3 implies that if $k \equiv 3 \bmod 4, k>3$, and $\mathfrak{n}$ is the normal bundle to an embedding of $P_{k-1}$ into $R_{2 k-3}$, which exists by [4], then $\mathfrak{n} \otimes H$ has a cross section. Hence $H_{k-1} \subset R^{2 k-3}$ and (of course) $P_{0} \subset S^{0}$. Now 7.2.2(a) follows from 7.2.1 and the remark following the proof of 7.2.1.

THEOREM 7.2.5. Let $\mathfrak{b}$ be an orientable $k-1$ plane bundle over $P_{k}$. If $w_{2}(\mathfrak{b})=0, w_{k-2}(\mathfrak{b})=0, k \equiv 0 \bmod 2$, and $k>5$, then $\mathfrak{b}^{2}$ has a cross section.

Proof. Suppose first that $k \equiv 0 \bmod 4$ and let $n=k-3$. In this case $\mathfrak{b}^{2}$ is a $V_{n+2,2}$ bundle where $n \equiv 1 \bmod 4$. As before there exists a bundle $\mathfrak{a}$ over $P_{k+1}$ and an integer $s$ such that $\mathfrak{b} \oplus s I=i^{-1} \mathfrak{a}$ where $i$ is the inclusion map $P_{k}<P_{k+1}$. This representation gives us a particular cross section of $\mathfrak{a}^{s}$ over the $k$-skeleton of $P_{k+1}$. The obstruction cocycle for extending this cross section lies in $\mathscr{Z}^{k+1}\left(P_{k+1} ; \pi_{k}\left(V_{k-1+s, s}\right)\right)=0$ since $\pi_{k}\left(V_{k-1+s, s}\right)=0$ is $s \geqq 3($ and $k \equiv 0 \bmod 4)$. Therefore we can suppose $\mathfrak{b}$ is a $k-1$ plane bundle over $P_{k+1}$.

Consider the following diagram

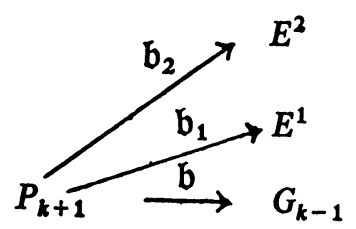

The only obstruction to finding $\mathfrak{b}_{1}$ is $w_{k-2}(\mathfrak{b})$, and this is zero by hypothesis. Now the indeterminacy of $b_{1}^{*} \mathbf{k}_{2}^{2}$ is all of $H^{k}\left(P_{k+1} ; Z_{2}\right)$ so we can choose $b_{1}$ so that $\mathfrak{b}_{1}^{*} \mathbf{k}_{2}^{2}=0$. The indeterminacy of $\mathfrak{b}_{1}^{*} \mathbf{k}_{1}^{2}$ is zero which means that different choices of $\mathfrak{b}_{1}$ do not affect $\mathfrak{b}_{1}^{*} \mathbf{k}_{1}^{2}$. On the other hand Theorem 6.3.1,(b) implies $S q^{2} \mathfrak{b}^{*} \mathbf{k}_{1}^{2}+S q^{1} \mathfrak{b}_{1}^{*}\left(\mathbf{k}_{2}^{2}\right)=0=S q^{2} \mathfrak{b}_{1}^{*}\left(\mathbf{k}_{1}^{2}\right)$. In the dimension involved $S q^{2}$ is an isomorphism, hence $\mathfrak{b}_{1}^{*}\left(\mathbf{k}_{1}^{2}\right)=0$. Therefore the map $\mathfrak{b}_{2}$ can be defined for a particu- 
lar choice of $\mathfrak{b}_{1}$. Now the indeterminacy of $\mathfrak{b}_{2}^{*} \mathbf{k}_{1}^{3}$ includes $S q^{1} H^{k-1}\left(P_{k+1} ; Z_{2}\right)$ and this is all of $H^{k}\left(P_{k+1} ; Z_{2}\right)$, therefore $\mathfrak{b}_{2}$ can be chosen so that $b_{2}^{*} k_{1}^{3}=0$ and hence $\mathfrak{b}^{2}$ has a cross section over $P_{k}$.

Suppose now that $k \equiv 2 \bmod 4$. In this case $\mathbf{b}^{2}$ is a $V_{n+2,2}$ bundle $(n=k-3)$ where $n \equiv 3 \bmod 4$. Hence the indeterminacy of $\mathbf{k}_{1}^{2}$ is all of $H^{k-1}\left(P_{k} ; Z_{2}\right)$ while the indeterminacy of $\mathbf{k}_{2}^{2}$ is zero. So a choice of $\mathfrak{b}_{1}$ can be made so that $\mathfrak{b}_{1}^{*} \mathbf{k}_{1}^{2}=0$ and $\mathfrak{b}_{1}^{*} \mathbf{k}_{2}^{2}$ is a single class independent of the choice of $\mathfrak{b}_{1}$. As in 7.2.4 we can find a bundle $\mathfrak{a}$ over $P_{k+2}$ and an integer $s$ such that $\mathfrak{b} \oplus s I=i^{-1} \mathfrak{a}$ where $i: P_{k} \subset P_{k+2}$. We have

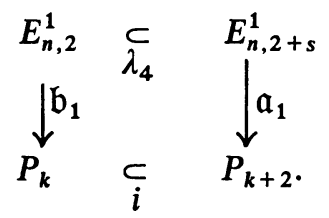

Now, using Lemma $6.5 .8, \mathfrak{b}_{1}^{*}\left({ }_{2} \mathbf{k}_{2}^{2}\right)=i^{*} \mathfrak{a}_{1}^{*}\left({ }_{m} \mathbf{k}_{2}^{2}\right)$ where $m=2+s$. By Theorem 6.3.1(f) we have $S q^{2} a_{1}^{*}\left({ }_{m} \mathbf{k}_{2}^{2}\right)=0$. But $S q^{2}$ in this dimension in $H^{*}\left(P_{k+2}\right)$ is an isomorphism, and therefore, $\mathfrak{a}_{1}^{*}\left({ }_{m} \mathbf{k}_{2}^{2}\right)=0$. This implies $\mathfrak{b}_{1}\left({ }_{2} \mathbf{k}_{2}^{2}\right)=0$ and the proof is completed as in the case $n=1 \bmod 4$.

We will now prove part (b) of 7.2.2. By the theorem of Epstein and Schwarzenberger [4], if $k \equiv 0 \bmod 2$ and $k \neq 2^{j}$, then $P_{k} \subset R^{2 k-1}$. Let $\mathfrak{n}$ be the normal bundle to this embedding. Then $\mathfrak{n} \otimes H$ satisfies the hypothesis of Theorem 7.2.5 except when $k=2^{j}-2$. Theorem 7.2.1, 7.2.5 and the remark following 7.2.1 then prove part (b) of 7.2.2, using the fact that $P_{1} \simeq S^{1}$.

7.2.8. We now prove part (c). Suppose $k \equiv 1 \bmod 4$ and $k \neq 2^{j}+1$. Then $P_{k-2} \subset P_{k-1} \subset R^{2 k-5}$ using part (b) of 7.2.2. Let $\mathfrak{n}$ be the normal bundle of $P_{k-2} \subset R^{2 k-5}$ and let $E(\mathfrak{n})$ be the total space of $\mathfrak{n}$. Then $E(\mathfrak{n}) \cap P_{k-1}$ is just $H_{k-2}$; hence $\mathfrak{n}=\mathfrak{n}^{\prime} \oplus H$. Then $\mathfrak{n} \otimes H=\left(\mathfrak{n}^{\prime} \otimes H\right) \oplus I$. Now $\mathfrak{n}^{\prime} \otimes H$ is a $k-4$ bundle over $P_{k-2}$ and $k-2=3 \bmod 4$. Hence Theorem 7.2.3 applies giving a cross section. Therefore $2 H_{k-2} \subset E(\mathfrak{n})$ and 7.2.1 now implies $P_{k} \subset R^{2 k-3}$ as before.

\section{REFERENCES}

1. J. F. Adams, On the non-existence of elements of Hopf invariant one, Ann. of Math. (2) 72 (1960), 20-104.

2. J. Adem, The iteration of the Steenrod squares in algebraic topology, Proc. Nat. Acad. Sci. U.S.A. 38 (1952), 720-726.

3. A. Borel, La cohomologie mod 2 de certains espaces homogenes, Comment. Math. Helv. 27 (1953), 165-197.

4. D. Epstein and R. Schwarzenberger, Imbeddings of real projective spaces, Ann. of Math. (2) 76 (1962), 180-184.

5. A. Haefliger, Plongements différentiables de variétés dans variétés, Comment. Math. Helv. 36 (1961), 47-82.

6. R. Hermann, Secondary obstructions for fiber spaces, Bull. Amer. Math. Soc. 65 (1959), 5-8. 
7. M Hirsch, Immersion of manifolds, Trans. Amer. Math. Soc. 93 (1959), 242-276.

8. S. T. Hu, Homotopy theory, Academic Press, New York, 1959.

9. S. D. Liao, On the theory of obstructions in fiber bundles, Ann. of Math. (2) 60 (1954), 146-191.

10. M. Mahowald, On extending cross sections in orientable $V_{k+m . m}$ bundles, Bull. Amer. Math. Soc. 68 (1962), 596-602.

11. W. Massey, Normal vector fields on manifolds, Proc. Amer. Math. Soc. 12 (1961), 33-40.

12. W. Massey and F. Peterson, On the dual Stiefel-Whitney classes of a manifold, (submitted to Bol. Soc. Mat. Mex.).

13. J. Milnor, Construction of universal bundles. I, Ann. of Math. (2) 63 (1956), 272-284.

14. - Lectures on characteristic classes, Mimeographed notes, Princeton Univ., Princeton, N. J.

15. G. Paechter, The groups $\pi_{r}\left(V_{n, m}\right)$. I-V, Quart.J. Math. Oxford Ser. (2) 7 (1956), 249-268; 9 (1958), 8-27; 10 (1959), 17-37, 241-60; 11 (1960), 1-16.

16. N. Steenrod, The topology of fiber bundles, Princeton Univ. Press, Princeton, N. J., 1951.

17. W. T. Wu, Classes caractéristiques et i-carrés d'une variété, C. R. Acad. Sci. Paris 230 (1950), 508-511.

SYRACUSE UNIVERSITY,

SYRACUSE, New YoRK 